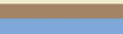

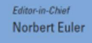

Journal of Nonlinear Mathematical Physics

ISSN (Online): 1776-0852 ISSN (Print): 1402-9251

Journal Home Page: https://www.atlantis-press.com/journals/jnmp

\title{
The Modified Korteweg-de Vries Equation on the Half-Line with a Sine-Wave as Dirichlet Datum
}

Guenbo Hwang, A. S. Fokas

To cite this article: Guenbo Hwang, A. S. Fokas (2013) The Modified Korteweg-de Vries Equation on the Half-Line with a Sine-Wave as Dirichlet Datum, Journal of Nonlinear Mathematical Physics 20:1, 135-157, DOI:

https://doi.org/10.1080/14029251.2013.792492

To link to this article: https://doi.org/10.1080/14029251.2013.792492

Published online: 04 January 2021 


\title{
The Modified Korteweg-de Vries Equation on the Half-Line with a Sine-Wave as Dirichlet Datum
}

\author{
Guenbo Hwang \\ Department of Mathematics, Daegu University, \\ Gyeonsan Gyeongbuk 712-714, Korea \\ ghwang@daegu.ac.kr \\ A. S. Fokas \\ Department of Applied Mathematics and Theoretical Physics, \\ University of Cambridge, Cambridge CB3 OWA, UK \\ t.fokas@damtp.cam.ac.uk \\ Received 14 January 2013 \\ Accepted 15 February 2013
}

\begin{abstract}
Boundary value problems for integrable nonlinear evolution PDEs, like the modified KdV equation, formulated on the half-line can be analyzed by the so-called unified transform method. For the modified KdV equation, this method yields the solution in terms of the solution of a matrix Riemann-Hilbert problem uniquely determined in terms of the initial datum $q(x, 0)$, as well as of the boundary values $\left\{q(0, t), q_{x}(0, t), q_{x x}(0, t)\right\}$. For the Dirichlet problem, it is necessary to characterize the unknown boundary values $q_{x}(0, t)$ and $q_{x x}(0, t)$ in terms of the given data $q(x, 0)$ and $q(0, t)$. It is shown here that in the particular case of a vanishing initial datum and of a sine wave as Dirichlet datum, $q_{x}(0, t)$ and $q_{x x}(0, t)$ can be computed explicitly at least up to third order in a perturbative expansion and that at least up to this order, these functions are asymptotically periodic for large $t$.
\end{abstract}

Keywords: Initial-boundary value problem; Generalized Dirichlet to Neumann map; modified Korteweg-de Vries equation.

2000 Mathematics Subject Classification: 47K15, 35Q55

\section{Introduction}

A unified method for analyzing boundary value problems for linear and integrable nonlinear PDEs was introduced in [9] and used extensively in the literature (reviews for the implementation of this method can be found in $[10,13,17])$. For integrable nonlinear evolution PDEs, the unified transform method yields novel integral representations formulated in the complex $k$-plane (the Fourier plane). These integrals, in addition to the exponentials which appear in the integrals of the linearized version of these nonlinear PDEs, also contain the entries of a matrix-valued function $M(x, t, k)$, which is the solution of a matrix Riemann-Hilbert $(\mathrm{RH})$ problem. This $\mathrm{RH}$ problem involves a jump matrix with explicit exponential $(x, t)$ dependence. In addition to explicit exponentials, the entries of the above jump matrix involve functions -called spectral functions- which depend only on $k$ and which can be computed in terms of the initial condition and the associated boundary values. For example, for the modified $\mathrm{KdV}$ equation on the half-line, the spectral functions can be computed in terms of $q(x, 0), q(0, t), q_{x}(0, t)$ and $q_{x x}(0, t)$; however for the Dirichlet problem $q_{x}(0, t)$ and $q_{x x}(0, t)$ are 
unknown. The most difficult step of the new method is the characterization of the unknown boundary values in terms of the given initial and boundary conditions. In a recent important development, this characterization was achieved via the analysis of the so-called global relation using two different approaches $[15,18]$. Both these approaches involve the analysis of a system of quadratically non-linear equations; the formulation in [15] is based on the direct analysis of the eigenfunctions characterizing the relevant spectral functions, while the formulation in [18] is based on an extension of the approach of [1]. However, it should be noted that: $(a)$ For a particular class of boundary conditions called linearizable, the global relation yields the spectral functions directly in terms of the given initial and boundary conditions bypassing the need to solve the above nonlinear equations. Thus, for this class of problems the new method is as effective as the classical inverse scattering transform method. (b) For the case that the boundary conditions vanish for large $t$, it is possible to obtain effective asymptotic results using the Deift-Zhou and the Deift-Zhou-Venakides techniques for the asymptotic analysis of these RH problems [5-8].

In contrast to the case that the boundary conditions vanish as $t \rightarrow \infty$, the analysis of the physically significant case of boundary conditions which are periodic in $t$, require the explicit characterization of the unknown boundary values in terms of the given data. Important results for such boundary value problems have been obtained in [2-4].

Here we concentrate on the simple example

$$
q(x, 0)=0, \quad 0<x<\infty ; \quad q(0, t)=\varepsilon \sin t+O\left(\varepsilon^{4}\right), \quad \varepsilon \rightarrow 0, \quad t>0 .
$$

Following the technique developed in [18] we compute explicitly the perturbation expansion in $\varepsilon$ of both $q_{x}(0, t)$ and $q_{x x}(0, t)$. These explicit formulae show that at least up to the third order in $\varepsilon$, both $q_{x}(0, t)$ and $q_{x x}(0, t)$ are asymptotically periodic as $t \rightarrow \infty$.

In a recent breakthrough $[14,16]$, it has been shown that in the case of the nonlinear Schrödinger equation, if

$$
q(x, 0)=0, \quad 0<x<\infty, \quad q(0, t)=a e^{i \omega t}+o(1), \quad t \rightarrow \infty,
$$

with $a, \omega$ real constants, then it is possible to solve the global relation exactly as $t \rightarrow \infty$ and to obtain an explicit formula for $q_{x}(0, t)$ as $t \rightarrow \infty$. The generalization of this result to the more complicated case of

$$
q(0, t)=a \sin \omega t+o(t), \quad t \rightarrow \infty,
$$

for both the nonlinear Schrödinger and the modified KdV equations is an open problem.

\section{The spectral functions of the $\mathrm{mKdV}$ equation}

We study the $\mathrm{mKdV}$ equation on the half-line

$$
q_{t}+q_{x x x}-6 \lambda q^{2} q_{x}=0, \quad 0<x<\infty, \quad 0<t<T,
$$

where $\lambda= \pm 1, T<\infty$ and $q(x, t)$ decays rapidly for all $t$ as $x \rightarrow \infty$. We assume that the given initial condition $q_{0}(x)=q(x, 0)$ vanishes sufficiently fast for all $t$ as $x \rightarrow \infty$. We denote the boundary values as

$$
g_{0}(t)=q(0, t), \quad g_{1}(t)=q_{x}(0, t), \quad g_{2}(t)=q_{x x}(0, t),
$$

which are assumed to be sufficiently smooth. 
It is well-known that (2.1) admits the following Lax pair formulation:

$$
\begin{aligned}
\mu_{x}+i f_{1}(k) \hat{\sigma}_{3} \mu & =Q(x, t, k) \mu, \\
\mu_{t}+i f_{2}(k) \hat{\sigma}_{3} \mu & =\tilde{Q}(x, t, k) \mu,
\end{aligned}
$$

where $k \in \mathbb{C}$ is a spectral parameter, $\mu$ is a $2 \times 2$ matrix-valued eigenfunction, $\hat{\sigma}_{3} A=\left[\sigma_{3}, A\right]$ with $\sigma_{3}=\operatorname{diag}(1,-1)$, and

$$
\begin{aligned}
& f_{1}(k)=k, \quad f_{2}(k)=4 k^{3}, \quad Q(x, t)=\left(\begin{array}{cc}
0 & q \\
\lambda & q
\end{array}\right), \\
& \tilde{Q}(x, t)=2 Q^{3}-Q_{x x}-2 i k\left(Q^{2}+Q_{x}\right) \sigma_{3}+4 k^{2} Q .
\end{aligned}
$$

Let the domains $\left\{D_{j}\right\}_{1}^{4}$ be given by (cf. figure 1)

$$
\begin{array}{ll}
D_{1}=\left\{\operatorname{Im} f_{1}>0 \text { and } \operatorname{Im} f_{2}>0\right\}, & D_{2}=\left\{\operatorname{Im} f_{1}>0 \text { and } \operatorname{Im} f_{2}<0\right\}, \\
D_{3}=\left\{\operatorname{Im} f_{1}<0 \text { and } \operatorname{Im} f_{2}>0\right\}, & D_{4}=\left\{\operatorname{Im} f_{1}<0 \text { and } \operatorname{Im} f_{2}<0\right\} .
\end{array}
$$

We introduce the complex-valued functions $\Psi(x, k)$ and $\Phi(x, k)$ as the following solutions of the $x$-part and the $t$-part of the Lax pair (2.3) evaluated at $t=0$ and $x=0$, respectively:

$$
\begin{array}{rlrl}
\Psi_{x}+i f_{1}(k) \hat{\sigma}_{3} \Psi=Q_{0} \Psi, & \lim _{x \rightarrow \infty} \Psi=I, \\
\Phi_{x}+i f_{2}(k) \hat{\sigma}_{3} \Phi & =\tilde{Q}_{0} \Psi, & \Phi(0, t)=I,
\end{array}
$$

where $Q_{0}(x)=Q(x, 0)$ and $\tilde{Q}_{0}(t, k)=\tilde{Q}(0, t, k)$. Equations (2.8) imply that the functions $\Psi$ and $\Phi$ satisfy the following system of linear Volterra integral equations:

$$
\begin{aligned}
& \Psi(x, k)=I-\int_{x}^{\infty} e^{-i f_{1}(k)(x-\xi) \hat{\sigma}_{3}}\left(Q_{0} \Psi\right)(\xi, k) d \xi, \quad k \in\left(\mathbb{C}^{-}, \mathbb{C}^{+}\right), \\
& \Phi(x, k)=I+\int_{0}^{t} e^{-i f_{2}(k)(t-\tau) \hat{\sigma}_{3}}\left(\tilde{Q}_{0} \Phi\right)(\tau, k) d \tau, \quad k \in \mathbb{C},
\end{aligned}
$$

where the notation $k \in\left(\mathbb{C}^{-}, \mathbb{C}^{+}\right)$in (2.9) denotes that the first and second column vectors of $\Psi(x, k)$ are defined for $\operatorname{Im} k \leq 0$ and $\operatorname{Im} k \geq 0$ respectively. Due to the symmetry of $Q$, the functions $\Psi$ and $\Phi$ can be written in the form

$$
\begin{aligned}
& \Psi(x, k)=\left(\begin{array}{cc}
\overline{\Psi_{2}(x, \bar{k})} & \Psi_{1}(x, k) \\
\lambda \overline{\Psi_{1}(x, \bar{k})} & \Psi_{2}(x, k)
\end{array}\right), \quad k \in\left(\mathbb{C}^{-}, \mathbb{C}^{+}\right), \\
& \Phi(t, k)=\left(\begin{array}{cc}
\overline{\Phi_{2}(t, \bar{k})} & \Phi_{1}(t, k) \\
\lambda \overline{\Phi_{1}(t, \bar{k})} & \Phi_{2}(t, k)
\end{array}\right), \quad k \in \mathbb{C} .
\end{aligned}
$$

The spectral functions $\{a(k), b(k), A(k), B(k)\}$ can be expressed in terms of the initial datum and of the boundary values. Given $q_{0}(x)$, the spectral functions $\{a(k), b(k)\}$ are defined by

$$
a(k)=\Psi_{2}(0, k), \quad b(k)=\Psi_{1}(0, k), \quad k \in \mathbb{C}^{+},
$$

while the spectral functions $\{A(k), B(k)\}$ are defined by

$$
A(k)=\overline{\Phi_{2}(T, \bar{k})}, \quad B(k)=-e^{2 i f_{2}(k) T \sigma_{3}} \Phi_{1}(T, k), \quad k \in \mathbb{C},
$$

in terms of $\left\{g_{0}, g_{1}, g_{2}\right\}$. 
The spectral functions satisfy the global relation

$$
\Phi_{1}(t, k)+\frac{b(k)}{a(k)} \overline{\Phi_{2}(t, \bar{k})} e^{-2 i f_{2}(k) t}=c(t, k), \quad \operatorname{Im} k \geq 0,
$$

where $c(t, k)$ is analytic and bounded in $\operatorname{Im} k>0$ and is of $O(1 / k)$ as $k \rightarrow \infty$.

It has been shown in $[12,15]$ that the functions $\Phi_{1}$ and $\Phi_{2}$ satisfy the following system of nonlinear integral equations:

$$
\begin{aligned}
& \Phi_{1}(t, k)=\int_{0}^{t} e^{-8 i k^{3}\left(t-t^{\prime}\right)}\left[-2 i k \lambda g_{0}^{2} \Phi_{1}+\left(2 \lambda g_{0}^{3}+4 k^{2} g_{0}+2 i k g_{1}-g_{2}\right) \Phi_{2}\right]\left(t^{\prime}, k\right) d t^{\prime} \\
& \Phi_{2}(t, k)=1+\lambda \int_{0}^{t}\left[\left(2 \lambda g_{0}^{3}+4 k^{2} g_{0}-2 i k g_{1}-g_{2}\right) \Phi_{1}+2 i k g_{0}^{2} \Phi_{2}\right]\left(t^{\prime}, k\right) d t^{\prime}
\end{aligned}
$$

Furthermore, for the Dirichlet problem, the unknown boundary values $g_{1}(t)$ and $g_{2}(t)$ satisfy the following relations:

$$
\begin{aligned}
g_{1}(t)= & \frac{2 g_{0}(t)}{\pi} \int_{\partial D_{3}} \chi_{2}(t, k) d k+\frac{2}{\pi i} \int_{\partial D_{3}}\left[k \chi_{1}(t, k)-\frac{3 g_{0}(t)}{2 i}\right] d k \\
& -\frac{2}{\pi i} \int_{\partial D_{3}} k e^{-8 i k^{3} t}\left[\left(\alpha^{2}-\alpha\right) \frac{b(\alpha k)}{a(\alpha k)} \overline{\Phi_{2}(t, \overline{\alpha k})}+\left(\alpha-\alpha^{2}\right) \frac{\left.b\left(\alpha^{2} k\right) \overline{a\left(\alpha^{2} k\right)} \overline{\Phi_{2}\left(t, \overline{\alpha^{2} k}\right)}\right] d k}{}\right. \\
& +4\left\{\left(1-\alpha^{2}\right) \sum_{k_{j} \in D_{1}^{\prime}}+(1-\alpha) \sum_{k_{j} \in D_{1}^{\prime \prime}}\right\} k_{j} e^{-8 i k_{j}^{3} t} \underset{k_{j}}{\operatorname{Res} \frac{b(k)}{a(k)} \overline{\Phi_{2}(t, \bar{k})}}
\end{aligned}
$$

and

$$
\begin{aligned}
g_{2}(t)= & \lambda g_{0}^{3}(t)-\frac{4}{\pi} \int_{\partial D_{3}}\left[k^{2} \chi_{1}(t, k)-\frac{3 k g_{0}(t)}{2 i}\right] d k \\
& +\frac{4 g_{0}(t)}{\pi i} \int_{\partial D_{3}} k \hat{\chi}_{2}(t, k) d k+\frac{2 g_{1}(t)}{\pi} \int_{\partial D_{3}} \chi_{2}(t, k) d k \\
& +\frac{4}{\pi} \int_{\partial D_{3}} k^{2} e^{-8 i k^{3} t}\left[(1-\alpha) \frac{b(\alpha k)}{a(\alpha k)} \overline{\Phi_{2}(t, \overline{\alpha k})}+\left(1-\alpha^{2}\right) \frac{\left.b\left(\alpha^{2} k\right) \overline{a\left(\alpha^{2} k\right)} \overline{\Phi_{2}\left(t, \overline{\alpha^{2} k}\right)}\right] d k}{}\right. \\
& -8 i\left\{(1-\alpha) \sum_{k_{j} \in D_{1}^{\prime}}+\left(1-\alpha^{2}\right) \sum_{k_{j} \in D_{1}^{\prime \prime}}\right\} k_{j}^{2} e^{-8 i k_{j}^{3} t} \operatorname{Res}_{k_{j}} \frac{b(k)}{a(k)} \overline{\Phi_{2}(t, \bar{k})}
\end{aligned}
$$

where $\alpha=e^{2 \pi i / 3},\left\{k_{j}\right\}_{1}^{N}$ is the set of the possible zeros of $a(k)$ (assumed to be a finite set of simple zeros), $D_{1}^{\prime}=D_{1} \cap\{\operatorname{Re} k>0\}$ and $D_{1}^{\prime \prime}=D_{1} \cap\{\operatorname{Re} k<0\}$, and for $j=1,2$, the symmetric combinations $\chi_{j}, \hat{\chi}_{j}$ and $\check{\chi}_{j}$ are defined by

$$
\begin{aligned}
& \chi_{j}(t, k)=\Phi_{j}(t, k)+\alpha \Phi_{j}(t, \alpha k)+\alpha^{2} \Phi_{j}\left(t, \alpha^{2} k\right), \\
& \hat{\chi}_{j}(t, k)=\Phi_{j}(t, k)+\alpha^{2} \Phi_{j}(t, \alpha k)+\alpha \Phi_{j}\left(t, \alpha^{2} k\right), \\
& \check{\chi}_{j}(t, k)=\Phi_{j}(t, k)+\Phi_{j}(t, \alpha k)+\Phi_{j}\left(t, \alpha^{2} k\right) .
\end{aligned}
$$




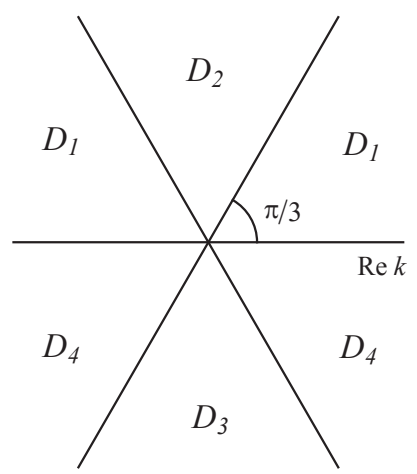

Fig. 1. The domains $\left\{D_{j}\right\}_{1}^{4}$.

\section{A perturbation expansion of the unknown boundary values}

We expand the functions $\Phi_{j}, q_{0}$ and $g_{0}$ as

$$
\begin{aligned}
\Phi_{j} & =\Phi_{j 0}+\varepsilon \Phi_{j 1}+\varepsilon^{2} \Phi_{j 2}+\cdots, \quad j=1,2, \\
q_{0} & =\varepsilon q_{01}+\varepsilon^{2} q_{02}+\cdots, \\
g_{0} & =\varepsilon g_{01}+\varepsilon^{2} g_{02}+\cdots,
\end{aligned}
$$

where $\varepsilon$ is a small perturbation parameter. Substituting (3.1a) into (2.15a) and (2.15b), we find $\Phi_{10}=0, \Phi_{20}=1$ and $\Phi_{21}=0$.

We next derive the first few terms of the above expansions. We assume a vanishing initial datum, that is, $q_{0}(x)=0$. In this case, $a(k)=1$ and $b(k)=0$.

Proposition 3.1. Assume that $q(x, 0)=0, x>0$, and

$$
q(0, t)=\varepsilon g_{01}+\varepsilon^{2} g_{02}+\varepsilon^{3} g_{03}+\cdots,
$$

where $\varepsilon>0$ is a small perturbation parameter and $g_{0 n}, n \geq 1$, are sufficiently smooth and compatible with the zero initial datum. (a) The unknown boundary value $q_{x}(0, t)$ can be expressed in the form

$$
q_{x}(0, t)=\varepsilon g_{11}(t)+\varepsilon^{2} g_{12}(t)+\varepsilon^{3} g_{13}(t)+\cdots,
$$

where

$$
g_{11}(t)=\frac{3 c_{1}}{\pi} \int_{0}^{t} \frac{\dot{g}_{01}\left(t^{\prime}\right)}{\left(t-t^{\prime}\right)^{1 / 3}} d t^{\prime}, \quad g_{12}(t)=\frac{3 c_{1}}{\pi} \int_{0}^{t} \frac{\dot{g}_{02}\left(t^{\prime}\right)}{\left(t-t^{\prime}\right)^{1 / 3}} d t^{\prime}
$$

and 


$$
\begin{aligned}
g_{13}( & (t)=-\frac{\lambda c_{1}}{\pi} \int_{0}^{t} g_{02}^{2}\left(t^{\prime}\right) \int_{0}^{t^{\prime}} \frac{g_{11}\left(t^{\prime \prime}\right)}{\left(t-t^{\prime \prime}\right)^{4 / 3}} d t^{\prime \prime} d t^{\prime}+\frac{3 c_{1}}{\pi} \int_{0}^{t} \frac{\dot{g}_{03}\left(t^{\prime}\right)}{\left(t-t^{\prime}\right)^{1 / 3}} d t^{\prime} \\
+ & \frac{\lambda c_{1}}{\pi}\left\{\int _ { 0 } ^ { t } g _ { 0 1 } ( t ^ { \prime } ) \left[\int_{0}^{t^{\prime}} g_{01}\left(t^{\prime \prime}\right) \int_{0}^{t^{\prime \prime}} \frac{\dot{g}_{11}\left(t^{\prime \prime \prime}\right)}{\tilde{t}^{4 / 3}} d t^{\prime \prime \prime} d t^{\prime \prime}-\int_{0}^{t^{\prime}} g_{11}\left(t^{\prime \prime}\right) \int_{0}^{t^{\prime \prime}} \frac{\dot{g}_{01}\left(t^{\prime \prime \prime}\right)}{\tilde{t}^{4 / 3}} d t^{\prime \prime \prime} d t^{\prime \prime}\right.\right. \\
& \left.-\int_{0}^{t^{\prime}} g_{21}\left(t^{\prime \prime}\right) \int_{0}^{t^{\prime \prime}} \frac{g_{21}\left(t^{\prime \prime \prime}\right)}{\tilde{t}^{4 / 3}} d t^{\prime \prime \prime} d t^{\prime \prime}\right] d t^{\prime}+\int_{0}^{t} g_{11}\left(t^{\prime}\right)\left[\int_{0}^{t^{\prime}} g_{01}\left(t^{\prime \prime}\right) \int_{0}^{t^{\prime \prime}} \frac{\dot{g}_{01}\left(t^{\prime \prime \prime}\right)}{\tilde{t}^{4 / 3}} d t^{\prime \prime \prime} d t^{\prime \prime}\right. \\
& \left.+\int_{0}^{t^{\prime}} g_{11}\left(t^{\prime \prime}\right) \int_{0}^{t^{\prime \prime}} \frac{g_{21}\left(t^{\prime \prime \prime}\right)}{\tilde{t}^{4 / 3}} d t^{\prime \prime \prime} d t^{\prime \prime}-\int_{0}^{t^{\prime}} g_{21}\left(t^{\prime \prime}\right) \int_{0}^{t^{\prime \prime}} \frac{g_{11}\left(t^{\prime \prime \prime}\right)}{\tilde{t}^{4 / 3}} d t^{\prime \prime \prime} d t^{\prime \prime}\right] d t^{\prime} \\
& -\int_{0}^{t} g_{21}\left(t^{\prime}\right)\left[\int_{0}^{t^{\prime}} g_{01}\left(t^{\prime \prime}\right) \int_{0}^{t^{\prime \prime}} \frac{g_{21}\left(t^{\prime \prime \prime}\right)}{\tilde{t}^{4 / 3}} d t^{\prime \prime \prime} d t^{\prime \prime}-\int_{0}^{t^{\prime}} g_{11}\left(t^{\prime \prime}\right) \int_{0}^{t^{\prime \prime}} \frac{g_{11}\left(t^{\prime \prime \prime}\right)}{\tilde{t}^{4 / 3}} d t^{\prime \prime \prime} d t^{\prime \prime}\right. \\
& \left.\left.+\int_{0}^{t^{\prime}} g_{21}\left(t^{\prime \prime}\right) \int_{0}^{t^{\prime \prime}} \frac{g_{01}\left(t^{\prime \prime \prime}\right)}{\tilde{t}^{4 / 3}} d t^{\prime \prime \prime} d t^{\prime \prime}\right] d t^{\prime}\right\}+\lambda g_{01}(t) \int_{0}^{t}\left[g_{11}^{2}-2 g_{01} g_{21}\right]\left(t^{\prime}\right) d t^{\prime},
\end{aligned}
$$

with $\tilde{t}=t-t^{\prime}+t^{\prime \prime}-t^{\prime \prime \prime}$ and

$$
c_{1}=-\frac{\sqrt{3}}{6} \Gamma(1 / 3)
$$

(b) The unknown boundary value $q_{x x}(0, t)$ can be expressed in the form

$$
q_{x x}(0, t)=\varepsilon_{21}(t)+\varepsilon^{2} g_{22}(t)+\varepsilon^{3} g_{23}(t)+\cdots,
$$

where

$$
g_{21}(t)=\frac{6 c_{2}}{i \pi} \int_{0}^{t} \frac{\dot{g}_{01}\left(t^{\prime}\right)}{\left(t-t^{\prime}\right)^{2 / 3}} d t^{\prime}, \quad g_{22}(t)=\frac{6 c_{2}}{i \pi} \int_{0}^{t} \frac{\dot{g}_{02}\left(t^{\prime}\right)}{\left(t-t^{\prime}\right)^{2 / 3}} d t^{\prime}
$$

and

$$
\begin{aligned}
g_{23} & (t)=\frac{3 \lambda}{2} g_{01}^{3}(t)-\frac{4 \lambda c_{2}}{\pi i} \int_{0}^{t} g_{02}^{2}\left(t^{\prime}\right) \int_{0}^{t^{\prime}} \frac{g_{11}\left(t^{\prime \prime}\right)}{\left(t-t^{\prime \prime}\right)^{5 / 3}} d t^{\prime \prime} d t^{\prime}+\frac{6 c_{2}}{\pi i} \int_{0}^{t} \frac{\dot{g}_{03}\left(t^{\prime}\right)}{\left(t-t^{\prime}\right)^{2 / 3}} d t^{\prime} \\
& +\frac{4 \lambda c_{2}}{\pi i}\left\{\int _ { 0 } ^ { t } g _ { 0 1 } ( t ^ { \prime } ) \left[\int_{0}^{t^{\prime}} g_{01}\left(t^{\prime \prime}\right) \int_{0}^{t^{\prime \prime}} \frac{\dot{g}_{11}\left(t^{\prime \prime \prime}\right)}{\tilde{t}^{5 / 3}} d t^{\prime \prime \prime} d t^{\prime \prime}-\int_{0}^{t^{\prime}} g_{11}\left(t^{\prime \prime}\right) \int_{0}^{t^{\prime \prime}} \frac{\dot{g}_{01}\left(t^{\prime \prime \prime}\right)}{\tilde{t}^{5 / 3}} d t^{\prime \prime \prime} d t^{\prime \prime}\right.\right. \\
& \left.-\int_{0}^{t^{\prime}} g_{21}\left(t^{\prime \prime}\right) \int_{0}^{t^{\prime \prime}} \frac{g_{21}\left(t^{\prime \prime \prime}\right)}{\tilde{t}^{5 / 3}} d t^{\prime \prime \prime} d t^{\prime \prime}\right] d t^{\prime}+\int_{0}^{t} g_{11}\left(t^{\prime}\right)\left[\int_{0}^{t^{\prime}} g_{01}\left(t^{\prime \prime}\right) \int_{0}^{t^{\prime \prime}} \frac{\dot{g}_{01}\left(t^{\prime \prime \prime}\right)}{\tilde{t}^{5 / 3}} d t^{\prime \prime \prime} d t^{\prime \prime}\right. \\
& \left.+\int_{0}^{t^{\prime}} g_{11}\left(t^{\prime \prime}\right) \int_{0}^{t^{\prime \prime}} \frac{g_{21}\left(t^{\prime \prime \prime}\right)}{\tilde{t}^{5 / 3}} d t^{\prime \prime \prime} d t^{\prime \prime}-\int_{0}^{t^{\prime}} g_{21}\left(t^{\prime \prime}\right) \int_{0}^{t^{\prime \prime}} \frac{g_{11}\left(t^{\prime \prime \prime}\right)}{\tilde{t}^{5 / 3}} d t^{\prime \prime \prime} d t^{\prime \prime}\right] d t^{\prime} \\
& -\int_{0}^{t} g_{21}\left(t^{\prime}\right)\left[\int_{0}^{t^{\prime}} g_{01}\left(t^{\prime \prime}\right) \int_{0}^{t^{\prime \prime}} \frac{g_{21}\left(t^{\prime \prime \prime}\right)}{\tilde{t}^{5 / 3}} d t^{\prime \prime \prime} d t^{\prime \prime}-\int_{0}^{t^{\prime}} g_{11}\left(t^{\prime \prime}\right) \int_{0}^{t^{\prime \prime}} \frac{g_{11}\left(t^{\prime \prime \prime}\right)}{\tilde{t}^{5 / 3}} d t^{\prime \prime \prime} d t^{\prime \prime}\right. \\
& \left.\left.+\int_{0}^{t^{\prime}} g_{21}\left(t^{\prime \prime}\right) \int_{0}^{t^{\prime \prime}} \frac{g_{01}\left(t^{\prime \prime \prime}\right)}{\tilde{t}^{5 / 3}} d t^{\prime \prime \prime} d t^{\prime \prime}\right] d t^{\prime}\right\}+\lambda g_{11}(t) \int_{0}^{t}\left[g_{11}^{2}-2 g_{01} g_{21}\right]\left(t^{\prime}\right) d t^{\prime},
\end{aligned}
$$

with

$$
c_{2}=\frac{i \sqrt{3}}{12} \Gamma(2 / 3)
$$


Proof. (a) Equations (2.16a) and (3.1) imply

$$
g_{11}(t)=\frac{2}{\pi i} \int_{\partial D_{3}}\left[k \chi_{11}(t, k)-\frac{3 g_{01}(t)}{2 i}\right] d k .
$$

The function $\Phi_{11}(t, k)$ is given by

$$
\Phi_{11}(t, k)=\int_{0}^{t} e^{-8 i k^{3}\left(t-t^{\prime}\right)}\left(4 k^{2} g_{01}+2 i k g_{11}-g_{21}\right)\left(t^{\prime}\right) d t^{\prime},
$$

and hence,

$$
\chi_{11}(t, k)=12 k^{2} \int_{0}^{t} e^{-8 i k^{3}\left(t-t^{\prime}\right)} g_{01}\left(t^{\prime}\right) d t^{\prime}
$$

Substituting (3.13) into (3.11) and then integrating by parts with respect to $d t^{\prime},(3.11)$ can be written in the form

$$
g_{11}(t)=\frac{3}{\pi} \int_{\partial D_{3}} \int_{0}^{t} e^{-8 i k^{3}\left(t-t^{\prime}\right)} \dot{g}_{01}\left(t^{\prime}\right) d t^{\prime} d k
$$

In order to integrate with respect $d k$, we will use the identity

$$
\int_{\partial D_{3}} e^{-8 i k^{3} t} d k=\frac{c_{1}}{t^{1 / 3}}, \quad c_{1}=-\frac{\sqrt{3}}{6} \Gamma(1 / 3) .
$$

In order to derive (3.15) we let $\partial D_{3}=C_{1} \cup C_{2}$, where $C_{1}=\partial D_{3} \cap \mathbb{C}_{\mathrm{IV}}$ and $C_{2}=\partial D_{3} \cap \mathbb{C}_{\mathrm{III}}$ (see figure 2). Cauchy's theorem implies

$$
\int_{C_{1}} e^{-8 i k^{3} t} d k=-\int_{\tilde{C}_{1}} e^{-8 i k^{3} t} d k=-e^{-i \pi / 6} \int_{0}^{\infty} e^{-8 \rho^{3} t} d \rho,
$$

where $\tilde{C}_{1}=\{\arg k=-i \pi / 6\}$ (cf. figure 2). Hence, evaluating the rhs of the above equation, we obtain

$$
\int_{C_{1}} e^{-8 i k^{3} t} d k=-\frac{e^{-i \pi / 6}}{6} \Gamma(1 / 3) \frac{1}{t^{1 / 3}}
$$

For the integral along $C_{2}$, we change variables, $k \rightarrow \alpha^{2} k$. Then the contour $C_{2}$ is deformed into $[0, \infty)$, and in analogy with (3.15) we now find

$$
\int_{C_{2}} e^{-8 i k^{3} t} d k=\frac{e^{-5 i \pi / 6}}{6} \Gamma(1 / 3) \frac{1}{t^{1 / 3}}
$$

Hence (3.17) and (3.18) imply (3.15). Using (3.15) in (3.14), we obtain

$$
g_{11}(t)=\frac{3 c_{1}}{\pi} \int_{0}^{t} \frac{\dot{g}_{0 j}\left(t^{\prime}\right)}{\left(t-t^{\prime}\right)^{1 / 3}} d t^{\prime}
$$

In a similar way we find that $g_{12}(t)$ is given by (3.4). 


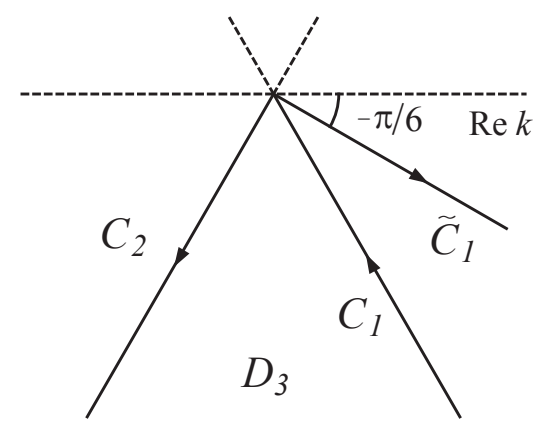

Fig. 2. The oriented contours $C_{1}, C_{2}$ and $\tilde{C}_{1}$.

In order to derive (3.5), we note that

$$
g_{13}(t)=\frac{2}{\pi i} \int_{\partial D_{3}}\left[k \chi_{13}(t, k)-\frac{3 g_{03}(t)}{2 i}\right] d k+\frac{2 g_{01}(t)}{\pi} \int_{\partial D_{3}} \chi_{22}(t, k) d k .
$$

Using (2.15b) and (3.1), we find that the function $\Phi_{22}$ is given by

$$
\Phi_{22}(t, k)=\lambda \int_{0}^{t}\left[\left(4 k^{2} g_{01}-2 i k g_{11}-g_{21}\right) \Phi_{11}+2 i k g_{01}^{2}\right] d t^{\prime} .
$$

Thus,

$$
\begin{aligned}
& \chi_{22}(t, k)=\lambda \int_{0}^{t}\left(4 k^{2} g_{01} \check{\chi}_{11}-2 i k g_{11} \hat{\chi}_{11}-g_{21} \chi_{11}\right) d t^{\prime}, \\
& \hat{\chi}_{22}(t, k)=\lambda \int_{0}^{t}\left(4 k^{2} g_{01} \chi_{11}-2 i k g_{11} \check{\chi}_{11}-g_{21} \hat{\chi}_{11}+6 i k g_{01}^{2}\right) d t^{\prime} \\
& \check{\chi}_{22}(t, k)=\lambda \int_{0}^{t}\left(4 k^{2} g_{01} \hat{\chi}_{11}-2 i k g_{11} \chi_{11}-g_{21} \check{\chi}_{11}\right) d t^{\prime},
\end{aligned}
$$

where the symmetric combinations $\hat{\chi}_{11}$ and $\check{\chi}_{11}$ are given by

$$
\begin{aligned}
& \hat{\chi}_{11}(t, k)=6 i k \int_{0}^{t} e^{-8 i k^{3}\left(t-t^{\prime}\right)} g_{11}\left(t^{\prime}\right) d t^{\prime}, \\
& \check{\chi}_{11}(t, k)=-3 \int_{0}^{t} e^{-8 i k^{3}\left(t-t^{\prime}\right)} g_{21}\left(t^{\prime}\right) d t^{\prime} .
\end{aligned}
$$

Furthermore, using (2.15a) we find

$$
\begin{aligned}
\Phi_{13}(t, k)= & \int_{0}^{t} e^{-8 i k^{3}\left(t-t^{\prime}\right)}\left[-2 i k \lambda g_{01}^{2} \Phi_{11}+\left(2 \lambda g_{01}^{3}+4 k^{2} g_{03}+2 i k g_{13}-g_{23}\right)\right. \\
& \left.+\left(4 k^{2} g_{01}+2 i k g_{11}-g_{21}\right) \Phi_{22}\right] d t^{\prime}
\end{aligned}
$$

which implies

$$
\chi_{13}(t, k)=\int_{0}^{t} e^{-8 i k^{3}\left(t-t^{\prime}\right)}\left[-2 i k \lambda g_{01}^{2} \hat{\chi}_{11}+12 k^{2} g_{03}+4 k^{2} g_{01} \check{\chi}_{22}+2 i k g_{11} \hat{\chi}_{22}-g_{21} \chi_{22}\right] d t^{\prime} .
$$


We next evaluate the second integral of (3.20); using (3.22) and (3.23) we obtain

$$
\begin{aligned}
\int_{\partial D_{3}} & \chi_{22}(t, k) d k=\lambda \int_{\partial D_{3}} 12 k^{2}\left\{\int _ { 0 } ^ { t } \left[-g_{01}\left(t^{\prime}\right) \int_{0}^{t^{\prime}} e^{-8 i k^{3}\left(t^{\prime}-t^{\prime \prime}\right)} g_{21}\left(t^{\prime \prime}\right) d t^{\prime \prime}\right.\right. \\
& \left.\left.+g_{11}\left(t^{\prime}\right) \int_{0}^{t^{\prime}} e^{-8 i k^{3}\left(t^{\prime}-t^{\prime \prime}\right)} g_{11}\left(t^{\prime \prime}\right) d t^{\prime \prime}-g_{21}\left(t^{\prime}\right) \int_{0}^{t^{\prime}} e^{-8 i k^{3}\left(t^{\prime}-t^{\prime \prime}\right)} g_{01}\left(t^{\prime \prime}\right) d t^{\prime \prime}\right] d t^{\prime}\right\} d k
\end{aligned}
$$

Using the identity

$$
\int_{\partial D_{3}} k^{2} \int_{0}^{t^{\prime}} e^{-8 i k^{3}\left(t^{\prime}-t^{\prime \prime}\right)} f\left(t^{\prime \prime}\right) d t^{\prime \prime} d k=\frac{\pi}{24} f\left(t^{\prime}\right)
$$

we can integrate the rhs of (3.26) with respect to $d k$ :

$$
\int_{\partial D_{3}} \chi_{22}(t, k) d k=\frac{\lambda \pi}{2} \int_{0}^{t} g_{11}^{2}\left(t^{\prime}\right) d t^{\prime}-\lambda \pi \int_{0}^{t} g_{01}\left(t^{\prime}\right) g_{21}\left(t^{\prime}\right) d t^{\prime} .
$$

Thus, the second integral of (3.20) yields

$$
\frac{g_{01}(t)}{\pi} \int_{\partial D_{3}} \chi_{22}(t, k) d k=\lambda g_{01}(t) \int_{0}^{t}\left[g_{11}^{2}-g_{01} g_{21}\right]\left(t^{\prime}\right) d t^{\prime} .
$$

Next we evaluate the first integral of (3.20); using (3.25) we find

$$
\begin{aligned}
\int_{\partial D_{3}} k \chi_{13}(t, k) d k= & \int_{\partial D_{3}} \int_{0}^{t} e^{-8 i k^{3}\left(t-t^{\prime}\right)}\left[-2 \lambda i k^{2} g_{01}^{2} \hat{\chi}_{11}+12 k^{3} g_{03}+4 k^{3} g_{01} \check{\chi}_{22}\right. \\
& \left.+2 i k^{2} g_{11} \hat{\chi}_{22}-k g_{21} \chi_{22}\right]\left(t^{\prime}, k\right) d t^{\prime} d k
\end{aligned}
$$

We will evaluate each integral of (3.30). For the first term of the rhs of (3.30), integrating by parts with respect to $d t^{\prime}$, we find

$$
\frac{3 \lambda}{2 i} \int_{\partial D_{3}} \int_{0}^{t} g_{02}^{2}\left(t^{\prime}\right)\left[e^{-8 i k^{3}\left(t-t^{\prime}\right)} g_{11}\left(t^{\prime}\right)-\int_{0}^{t^{\prime}} e^{-8 i k^{3}\left(t-t^{\prime \prime}\right)} \dot{g}_{11}\left(t^{\prime \prime}\right) d t^{\prime \prime}\right] d t^{\prime} d k .
$$

Using (3.15), (3.31) becomes

$$
\frac{3 \lambda c_{1}}{2 i} \int_{0}^{t} g_{02}^{2}\left(t^{\prime}\right) \frac{g_{11}\left(t^{\prime}\right)}{\left(t-t^{\prime}\right)^{1 / 3}} d t^{\prime}-\frac{3 \lambda c_{1}}{2 i} \int_{0}^{t} g_{02}^{2}\left(t^{\prime}\right) \int_{0}^{t^{\prime}} \frac{\dot{g}_{11}\left(t^{\prime \prime}\right)}{\left(t-t^{\prime \prime}\right)^{1 / 3}} d t^{\prime \prime} d t^{\prime}
$$

and then integrating by parts with respect to $d t^{\prime \prime}$, we obtain

$$
-2 \lambda i \int_{\partial D_{3}} k^{2} \int_{0}^{t} e^{-8 i k^{3}\left(t-t^{\prime}\right)} g_{01}^{2}\left(t^{\prime}\right) \hat{\chi}_{11}\left(t^{\prime}, k\right) d t^{\prime} d k=\frac{\lambda c_{1}}{2 i} \int_{0}^{t} g_{02}^{2}\left(t^{\prime}\right) \int_{0}^{t^{\prime}} \frac{g_{11}\left(t^{\prime \prime}\right)}{\left(t-t^{\prime \prime}\right)^{1 / 3}} d t^{\prime \prime} d t^{\prime} .
$$

Similarly, the second term of rhs of (3.30) yields

$$
\int_{\partial D_{3}} 12 k^{3} \int_{0}^{t} e^{-8 i k^{3}\left(t-t^{\prime}\right)} g_{03}\left(t^{\prime}\right) d t^{\prime} d k=\frac{3}{2 i} \int_{\partial D_{3}} g_{03}(t) d k-\frac{3 c_{1}}{2 i} \int_{0}^{t} \frac{\dot{g}_{03}\left(t^{\prime}\right)}{\left(t-t^{\prime}\right)^{1 / 3}} d t^{\prime}
$$


G. Hwang and A.S. Fokas

For the third term of the rhs of (3.30) we find

$$
\begin{array}{rl}
\int_{\partial D_{3}} & 4 k^{3} \int_{0}^{t} e^{-8 i k^{3}\left(t-t^{\prime}\right)} g_{01}\left(t^{\prime}\right) \check{\chi}_{22}\left(t^{\prime}, k\right) d t^{\prime} d k \\
& =\lambda i \int_{\partial D_{3}} 96 k^{6} \int_{0}^{t} g_{01}\left(t^{\prime}\right) \int_{0}^{t^{\prime}} g_{01}\left(t^{\prime \prime}\right) \int_{0}^{t^{\prime \prime}} e^{-8 i k^{3} \tilde{t}} g_{11}\left(t^{\prime \prime \prime}\right) d t^{\prime \prime \prime} d t^{\prime \prime} d t^{\prime} \\
& -\lambda i \int_{\partial D_{3}} 96 k^{6} \int_{0}^{t} g_{01}\left(t^{\prime}\right) \int_{0}^{t^{\prime}} g_{11}\left(t^{\prime \prime}\right) \int_{0}^{t^{\prime \prime}} e^{-8 i k^{3} \tilde{t}} g_{01}\left(t^{\prime \prime \prime}\right) d t^{\prime \prime \prime} d t^{\prime \prime} d t^{\prime} \\
& +\lambda \int_{\partial D_{3}} 12 k^{3} \int_{0}^{t} g_{01}\left(t^{\prime}\right) \int_{0}^{t^{\prime}} g_{21}\left(t^{\prime \prime}\right) \int_{0}^{t^{\prime \prime}} e^{-8 i k^{3} \tilde{t}} g_{21}\left(t^{\prime \prime \prime}\right) d t^{\prime \prime \prime} d t^{\prime \prime} d t^{\prime}
\end{array}
$$

where $\tilde{t}=t-t^{\prime}+t^{\prime \prime}-t^{\prime \prime \prime}$. Integrating by parts with respect to $d t^{\prime \prime \prime}$, the first integral of (3.35) becomes

$$
\begin{array}{rl}
\lambda \int_{\partial D_{3}} & 12 k^{3} \int_{0}^{t} e^{-8 i k^{3}\left(t-t^{\prime}\right)} g_{01}\left(t^{\prime}\right) \int_{0}^{t^{\prime}} g_{01}\left(t^{\prime \prime}\right) g_{11}\left(t^{\prime \prime}\right) d t^{\prime \prime} d t^{\prime} d k \\
& +\frac{3 \lambda i}{2} \int_{\partial D_{3}} \int_{0}^{t} e^{-8 i k^{3}\left(t-t^{\prime}\right)} g_{01}\left(t^{\prime}\right) \int_{0}^{t^{\prime}} g_{01}\left(t^{\prime \prime}\right) \dot{g}_{11}\left(t^{\prime \prime}\right)\left(t^{\prime \prime}\right) d t^{\prime \prime} d t^{\prime} d k \\
& -\frac{3 \lambda i}{2} \int_{\partial D_{3}} \int_{0}^{t} g_{01}\left(t^{\prime}\right) \int_{0}^{t^{\prime}} g_{01}\left(t^{\prime \prime}\right) \int_{0}^{t^{\prime \prime}} e^{-8 i k^{3} \ddot{t}_{11}} \ddot{g}_{11}\left(t^{\prime \prime}\right) d t^{\prime \prime} d t^{\prime} d k
\end{array}
$$

Integrating by parts the first term of the above expression with respect to $d t^{\prime}$ and then employing (3.15), we find

$$
\begin{array}{rl}
\lambda i \int_{\partial D_{3}} & 96 k^{6} \int_{0}^{t} g_{01}\left(t^{\prime}\right) \int_{0}^{t^{\prime}} g_{01}\left(t^{\prime \prime}\right) \int_{0}^{t^{\prime \prime}} e^{-8 i k^{3} t} g_{11}\left(t^{\prime \prime \prime}\right) d t^{\prime \prime \prime} d t^{\prime \prime} d t^{\prime} \\
& =\frac{3 \lambda}{2 i} \int_{\partial D_{3}} g_{01}(t) \int_{0}^{t} g_{01}\left(t^{\prime}\right) g_{11}\left(t^{\prime}\right) d t^{\prime}-\frac{3 \lambda c_{1}}{2 i} \int_{0}^{t} \frac{\dot{g}_{01}\left(t^{\prime}\right)}{\left(t-t^{\prime}\right)^{1 / 3}} \int_{0}^{t^{\prime}} g_{01}\left(t^{\prime \prime}\right) g_{11}\left(t^{\prime \prime}\right) d t^{\prime} \\
& -\frac{3 \lambda c_{1}}{2 i} \int_{0}^{t} \frac{g_{01}^{2}\left(t^{\prime}\right) g_{11}\left(t^{\prime}\right)}{\left(t-t^{\prime}\right)^{1 / 3}} d t^{\prime}-\frac{\lambda c_{1}}{2 i} \int_{0}^{t} g_{01}\left(t^{\prime}\right) \int_{0}^{t^{\prime}} g_{01}\left(t^{\prime}\right) \int_{0}^{t^{\prime \prime}} \frac{\dot{g}_{11}\left(t^{\prime \prime \prime}\right)}{\tilde{t}^{4 / 3}} d t^{\prime \prime} d t^{\prime}
\end{array}
$$

The other two terms of the rhs of (3.35) can be computed in a similar way; hence (3.35) becomes

$$
\begin{aligned}
\int_{\partial D_{3}} 4 k^{3} & \int_{0}^{t} e^{-8 i k^{3}\left(t-t^{\prime}\right)} g_{01}\left(t^{\prime}\right) \check{\chi}_{22}\left(t^{\prime}, k\right) d t^{\prime} d k \\
& =-\frac{\lambda c_{1}}{2 i} \int_{0}^{t} g_{01}\left(t^{\prime}\right)\left\{\int_{0}^{t^{\prime}} g_{01}\left(t^{\prime}\right) \int_{0}^{t^{\prime \prime}} \frac{\dot{g}_{11}\left(t^{\prime \prime \prime}\right)}{\tilde{t}^{4 / 3}} d t^{\prime \prime} d t^{\prime}\right. \\
& \left.-\int_{0}^{t^{\prime}} g_{11}\left(t^{\prime}\right) \int_{0}^{t^{\prime \prime}} \frac{\dot{g}_{01}\left(t^{\prime \prime \prime}\right)}{\tilde{t}^{4 / 3}} d t^{\prime \prime \prime} d t^{\prime \prime} d t^{\prime}-\int_{0}^{t^{\prime}} g_{21}\left(t^{\prime}\right) \int_{0}^{t^{\prime \prime}} \frac{g_{21}\left(t^{\prime \prime \prime}\right)}{\tilde{t}^{4 / 3}} d t^{\prime \prime \prime} d t^{\prime \prime} d t^{\prime}\right\}
\end{aligned}
$$

Similarly, the fourth term of (3.30) yields

$$
\begin{aligned}
2 i \int_{\partial D_{3}} k^{2} & \int_{0}^{t} e^{-8 i k^{3}\left(t-t^{\prime}\right)} g_{11}\left(t^{\prime}\right) \hat{\chi}_{22}\left(t^{\prime}, k\right) d t^{\prime} d k \\
& =-\frac{\lambda c_{1}}{2 i} \int_{0}^{t} g_{11}\left(t^{\prime}\right)\left\{\int_{0}^{t^{\prime}} g_{01}\left(t^{\prime}\right) \int_{0}^{t^{\prime \prime}} \frac{\dot{g}_{01}\left(t^{\prime \prime \prime}\right)}{\tilde{t}^{4 / 3}} d t^{\prime \prime \prime} d t^{\prime \prime} d t^{\prime}\right. \\
& \left.+\int_{0}^{t^{\prime}} g_{11}\left(t^{\prime}\right) \int_{0}^{t^{\prime \prime}} \frac{g_{21}\left(t^{\prime \prime \prime}\right)}{\tilde{t}^{4 / 3}} d t^{\prime \prime \prime} d t^{\prime \prime} d t^{\prime}-\int_{0}^{t^{\prime}} g_{21}\left(t^{\prime}\right) \int_{0}^{t^{\prime \prime}} \frac{g_{11}\left(t^{\prime \prime \prime}\right)}{\tilde{t}^{4 / 3}} d t^{\prime \prime \prime} d t^{\prime \prime} d t^{\prime}\right\},
\end{aligned}
$$


and the last integral of (3.30) becomes

$$
\begin{aligned}
-\int_{\partial D_{3}} k & \int_{0}^{t} e^{-8 i k^{3}\left(t-t^{\prime}\right)} g_{21}\left(t^{\prime}\right) \chi_{22}\left(t^{\prime}, k\right) d t^{\prime} d k \\
& =\frac{\lambda c_{1}}{2 i} \int_{0}^{t} g_{21}\left(t^{\prime}\right)\left\{\int_{0}^{t^{\prime}} g_{01}\left(t^{\prime}\right) \int_{0}^{t^{\prime \prime}} \frac{g_{21}\left(t^{\prime \prime \prime}\right)}{\tilde{t}^{4 / 3}} d t^{\prime \prime \prime} d t^{\prime \prime} d t^{\prime}\right. \\
& \left.-\int_{0}^{t^{\prime}} g_{11}\left(t^{\prime}\right) \int_{0}^{t^{\prime \prime}} \frac{g_{11}\left(t^{\prime \prime \prime}\right)}{\tilde{t}^{4 / 3}} d t^{\prime \prime \prime} d t^{\prime \prime} d t^{\prime}+\int_{0}^{t^{\prime}} g_{21}\left(t^{\prime}\right) \int_{0}^{t^{\prime \prime}} \frac{g_{01}\left(t^{\prime \prime \prime}\right)}{\tilde{t}^{4 / 3}} d t^{\prime \prime \prime} d t^{\prime \prime} d t^{\prime}\right\} .
\end{aligned}
$$

Combining (3.39) with (3.40), (3.33), (3.34) and (3.38), as well as (3.29), we obtain (3.5).

(b) Recall that $q_{x x}(0, t)=g_{2}(t)$ is given by

$$
\begin{aligned}
g_{2}(t)= & \lambda g_{0}^{3}(t)-\frac{4}{\pi} \int_{\partial D_{3}}\left[k^{2} \chi_{1}(t, k)-\frac{3 k g_{0}}{2 i}\right] d k \\
& +\frac{4 g_{0}(t)}{i \pi} \int_{\partial D_{3}} k \hat{\chi}_{2}(t, k) d k+\frac{2 g_{1}(t)}{\pi} \int_{\partial D_{3}} \chi_{2}(t, k) d k .
\end{aligned}
$$

Hence, the first term of the expansion of $g_{2}$ is

$$
g_{21}(t)=-\frac{4}{\pi} \int_{\partial D_{3}}\left[k^{2} \chi_{11}(t, k)-\frac{3 k g_{01}}{2 i}\right] d k
$$

Substituting (3.13) into (3.42) and integrating by parts with respect to $d t^{\prime}$ we find

$$
g_{21}(t)=\frac{6}{i \pi} \int_{\partial D_{3}} k \int_{0}^{t} e^{-8 i k^{3}\left(t-t^{\prime}\right)} \dot{g}_{01}\left(t^{\prime}\right) d t^{\prime} d k
$$

Following steps similar to those used in $(a)$, we find

$$
\int_{\partial D_{3}} k e^{-8 i k^{3} t} d k=\frac{c_{2}}{t^{2 / 3}}, \quad c_{2}=\frac{i \sqrt{3}}{12} \Gamma(2 / 3)
$$

Hence, employing (3.44) we obtain

$$
g_{21}(t)=\frac{6 c_{2}}{i \pi} \int_{0}^{t} \frac{\dot{g}_{01}\left(t^{\prime}\right)}{\left(t-t^{\prime}\right)^{2 / 3}} d t^{\prime}
$$

The function $g_{22}(t)$ can be obtained in a similar manner.

Next, we derive the third order term of the expansion, which satisfies

$$
\begin{aligned}
g_{23}(t)= & \lambda g_{01}^{3}-\frac{4}{\pi} \int_{\partial D_{3}}\left[k^{2} \chi_{13}(t, k)-\frac{3 k g_{01}}{2 i}\right] d k \\
& +\frac{4 g_{01}(t)}{i \pi} \int_{\partial D_{3}} k \hat{\chi}_{22}(t, k) d k+\frac{2 g_{11}(t)}{\pi} \int_{\partial D_{3}} \chi_{22}(t, k) d k .
\end{aligned}
$$


In order to evaluate the second integral of the rhs of (3.46), we use (3.22b) together with (3.13) and (3.23):

$$
\begin{array}{rl}
\int_{\partial D_{3}} & k \hat{\chi}_{22}(t, k) d k=\lambda \int_{\partial D_{3}} 48 k^{5} \int_{0}^{t} g_{01}\left(t^{\prime}\right) \int_{0}^{t^{\prime}} e^{-8 i k^{3}\left(t^{\prime}-t^{\prime \prime}\right)} g_{01}\left(t^{\prime \prime}\right) d t^{\prime \prime} d t^{\prime} d k \\
& +\lambda i \int_{\partial D_{3}} 6 k^{2} \int_{0}^{t} g_{11}\left(t^{\prime}\right) \int_{0}^{t^{\prime}} e^{-8 i k^{3}\left(t^{\prime}-t^{\prime \prime}\right)} g_{21}\left(t^{\prime \prime}\right) d t^{\prime \prime} d t^{\prime} d k \\
& -\lambda i \int_{\partial D_{3}} 6 k^{2} \int_{0}^{t} g_{21}\left(t^{\prime}\right) \int_{0}^{t^{\prime}} e^{-8 i k^{3}\left(t^{\prime}-t^{\prime \prime}\right)} g_{11}\left(t^{\prime \prime}\right) d t^{\prime \prime} d t^{\prime} d k+\lambda i \int_{\partial D_{3}} 6 k^{2} \int_{0}^{t} g_{01}^{2}\left(t^{\prime}\right) d t^{\prime} d k .
\end{array}
$$

Using (3.27), we find that the second and third integrals of the rhs of (3.47) vanish. For the first integral of the rhs of (3.47), integrating by parts with respect to $d t^{\prime}$ we find

$$
-6 \lambda i \int_{\partial D_{3}} k^{2} \int_{0}^{t} g_{01}^{2}\left(t^{\prime}\right) d t^{\prime} d k+6 \lambda i \int_{\partial D_{3}} k^{2} \int_{0}^{t} g_{01}\left(t^{\prime}\right) \int_{0}^{t^{\prime}} e^{-8 i k^{3}\left(t^{\prime}-t^{\prime \prime}\right)} \dot{g}_{01}\left(t^{\prime}\right) d t^{\prime \prime} d t^{\prime} d k .
$$

The first term of (3.48) cancels with the last term of the rhs of (3.47). Also, using (3.27), the second term of (3.48) yields

$$
\frac{\lambda i \pi}{4} \int_{0}^{t} g_{01}\left(t^{\prime}\right) \dot{g}_{01}\left(t^{\prime}\right) d t^{\prime}=\frac{\lambda i \pi}{8} g_{01}^{2}(t) .
$$

Thus, we find

$$
\frac{4 g_{01}(t)}{i \pi} \int_{\partial D_{3}} k \hat{\chi}_{22}(t, k) d k=\frac{\lambda}{2} g_{01}^{3}(t) .
$$

The first and third integrals of (3.46) can be evaluated in a similar way as in $(a)$ and hence (3.9) follows.

\section{Periodic boundary condition}

We next concentrate in the particular case that the Dirichlet datum is a sine wave.

Proposition 4.1. Let

$$
q(0, t)=\varepsilon \sin t+O\left(\varepsilon^{4}\right), \quad \varepsilon \rightarrow 0, \quad t>0 ; \quad q(x, 0)=0, \quad x \in \mathbb{R}^{+} .
$$

Then, $q_{x}(0, t)$ and $q_{x x}(0, t)$ are given by (3.3) and (3.7), where

$$
\begin{aligned}
& g_{12}(t)=g_{22}(t)=0, \\
& g_{11}(t)=\frac{1}{2}\left(\alpha e^{i t}+\alpha^{2} e^{-i t}\right)+\int_{\partial \tilde{D}_{3}} e^{-8 i k^{3} t} F\left(k^{3}\right) d k, \\
& g_{21}(t)=\frac{i}{2}\left(\alpha^{2} e^{i t}-\alpha e^{-i t}\right)-2 i \int_{\partial \tilde{D}_{3}} e^{-8 i k^{3} t} k F\left(k^{3}\right) d k,
\end{aligned}
$$

with $\alpha=e^{2 i \pi / 3}, \partial \tilde{D}_{3}$ is depicted in figure 3 and

$$
F\left(k^{3}\right)=\frac{12}{i \pi}\left(\frac{k^{3}}{8 k^{3}+1}-\frac{k^{3}}{8 k^{3}-1}\right) .
$$




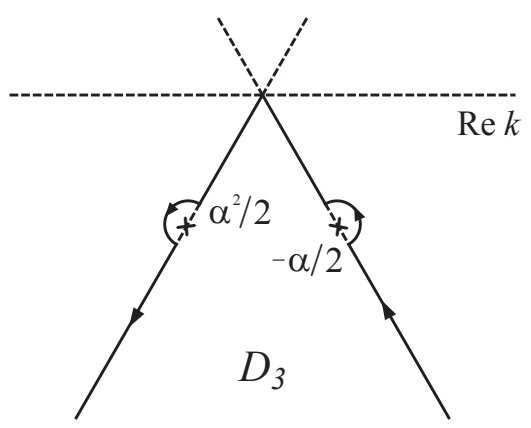

Fig. 3. The deformed contour $\partial \tilde{D}_{3}$, where $\alpha=e^{2 i \pi / 3}$.

Proof. Equation (3.13) implies that

$$
k \chi_{11}(t, k)=-6 i k^{3} e^{-8 i k^{3} t} \int_{0}^{t}\left(e^{i \tau\left(8 k^{3}+1\right)}-e^{i \tau\left(8 k^{3}-1\right)}\right) d \tau .
$$

Integrating the above equation with respect $d \tau$ and substituting the resulting expression into (3.11), we obtain

$$
g_{11}(t)=\frac{2}{i \pi} \int_{\partial D_{3}}\left[-\frac{6 k^{3}}{8 k^{3}+1}\left(e^{i t}-e^{-8 i k^{3} t}\right)+\frac{6 k^{3}}{8 k^{3}-1}\left(e^{-i t}-e^{-8 i k^{3} t}\right)+\frac{3}{4}\left(e^{i t}-e^{-i t}\right)\right] d k .
$$

Note that the integrand in (4.7) has removable singularities at $k=-\alpha / 2$ and at $k=\alpha^{2} / 2$. Hence, before splitting, the integral we deform the contour $\partial D_{3}$ to the contour $\partial \tilde{D}_{3}$ depicted in figure 3 . Thus, (4.7) yields

$$
g_{11}(t)=\frac{3}{2 i \pi} \int_{\partial \tilde{D}_{3}}\left(\frac{e^{i t}}{8 k^{3}+1}+\frac{e^{-i t}}{8 k^{3}-1}\right) d k+\frac{12}{i \pi} \int_{\partial \tilde{D}_{3}} e^{-8 i k^{3} t}\left(\frac{k^{3}}{8 k^{3}+1}-\frac{k^{3}}{8 k^{3}-1}\right) d k
$$

The first integral in (4.8) can be evaluated by the residue theorem, and hence we find $g_{11}(t)$ as in (4.3).

In order to derive (4.4) we use the representation

$$
g_{21}(t)=-\frac{3}{\pi} \int_{\partial \tilde{D}_{3}}\left(\frac{k e^{i t}}{8 k^{3}+1}+\frac{k e^{-i t}}{8 k^{3}-1}\right) d k-\frac{24}{\pi} \int_{\partial \tilde{D}_{3}} e^{-8 i k^{3} t}\left(\frac{k^{4}}{8 k^{3}+1}-\frac{k^{4}}{8 k^{3}-1}\right) d k .
$$

Proposition 4.2. Let $q(x, 0)$ and $q(0, t)$ be given by (4.1). Then the function $\Phi_{1}(t, k)$ defined by (2.15a) satisfies $\Phi_{1}(t, k)=\varepsilon \Phi_{11}(t, k)+O\left(\varepsilon^{2}\right), \varepsilon \rightarrow 0$, where

$$
\begin{aligned}
\Phi_{11}(t, k)= & \frac{e^{-8 i k^{3} t}-e^{i t}}{2(2 k+\alpha)}-\frac{e^{-8 i k^{3} t}-e^{-i t}}{2\left(2 k-\alpha^{2}\right)}+e^{-8 i k^{3} t}\left[\frac{\alpha(2 k-\alpha)}{2\left(8 k^{3}+1\right)}+\frac{\alpha^{2}\left(2 k+\alpha^{2}\right)}{2\left(8 k^{3}-1\right)}\right. \\
& \left.-\frac{4 k^{2}\left(\sigma^{2}+\sigma\right)}{\left(8 k^{3}+1\right)\left(8 k^{3}-1\right)}\right]+\frac{1}{4} \int_{\partial \tilde{D}_{3}^{\sigma k}} \frac{\left(k+k_{1}\right) F\left(k_{1}^{3}\right)}{k^{3}-k_{1}^{3}} e^{-8 i k_{1}^{3} t} d k_{1},
\end{aligned}
$$



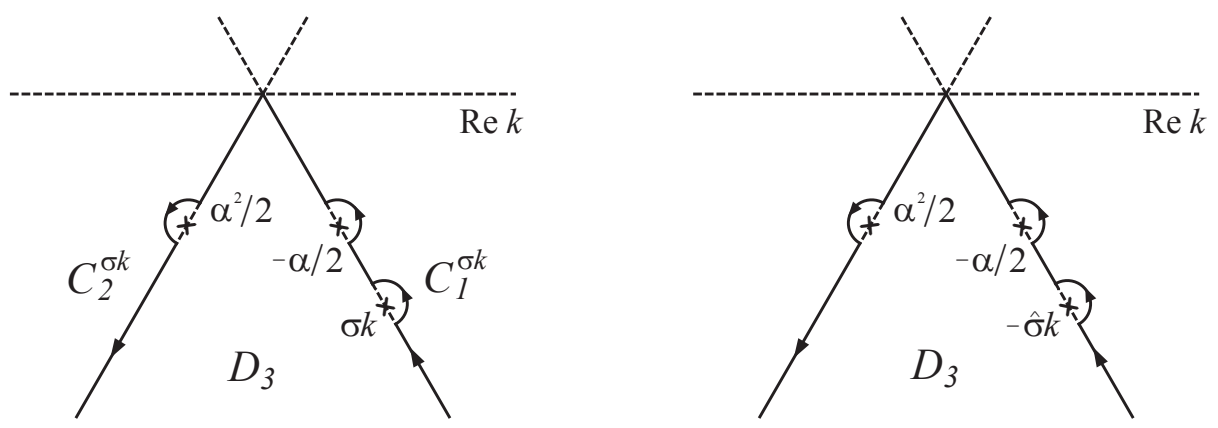

Fig. 4. The deformed contours $\partial \tilde{D}_{3}^{\sigma k}$ (left) and $\partial \tilde{D}_{3}^{\hat{\sigma} k}$ (right), where $\alpha=e^{2 i \pi / 3}$.

with $\partial \tilde{D}_{3}^{\sigma k}$ depicted in figure 4 , and $\sigma$ is given by

$$
\sigma= \begin{cases}1 & \text { if } k \in \partial D_{3} \\ \alpha & \text { if } \alpha k \in \partial D_{3} \\ \alpha^{2} & \text { if } \alpha^{2} k \in \partial D_{3}\end{cases}
$$

Proof. Substituting (4.3) and (4.4) into (3.12) and then integrating the resulting expression with respect to $d \tau$, we find

$$
\begin{aligned}
\Phi_{11}(t, k) & =\frac{e^{-8 i k^{3} t}-e^{i t}}{2\left(8 k^{3}+1\right)}\left(4 k^{2}-2 \alpha k+\alpha^{2}\right)-\frac{e^{-8 i k^{3} t}-e^{-i t}}{2\left(8 k^{3}-1\right)}\left(4 k^{2}+2 \alpha^{2} k+\alpha\right) \\
& +\frac{1}{4} \int_{\partial \tilde{D}_{3}} \frac{\left(k+k_{1}\right) F\left(k_{1}^{3}\right)}{k^{3}-k_{1}^{3}}\left(e^{-8 i k_{1}^{3} t}-e^{-8 i k^{3} t}\right) d k_{1}
\end{aligned}
$$

Employing the identities

$$
4 k^{2}-2 \alpha k+\alpha^{2}=(2 k+1)\left(2 k+\alpha^{2}\right), \quad 4 k^{2}+2 \alpha^{2} k+\alpha=(2 k-1)(2 k-\alpha),
$$

we obtain the first two terms in (4.10). On the other hand, the integrand in the second line in (4.12) has removable singularities at $k_{1}=k, \alpha k, \alpha^{2} k$. Hence, before splitting the integrals we deform the contours $\partial \tilde{D}_{3}$ to the contour $\partial \tilde{D}_{3}^{\sigma k}$ which are depicted in figure 4 .

Then, the residue theorem implies

$$
\int_{\partial \tilde{D}_{3}^{\sigma k}} \frac{\left(k+k_{1}\right) F\left(k_{1}^{3}\right)}{k^{3}-k_{1}^{3}} d k_{1}=-2\left[\frac{\alpha(2 k-\alpha)}{8 k^{3}+1}+\frac{\alpha^{2}\left(2 k+\alpha^{2}\right)}{8 k^{3}-1}-\frac{8 k^{2}\left(\sigma^{2}+\sigma\right)}{\left(8 k^{3}+1\right)\left(8 k^{3}-1\right)}\right]
$$

where $\sigma$ is given in (4.11). Using the above equation in (4.12), we find (4.10). 
Proposition 4.3. Let $q(x, 0)$ and $q(0, t)$ be given by (4.1). Then, $\Phi_{2}(t, k)$ defined by (2.15b) satisfies $\Phi_{2}(t, k)=1+\varepsilon^{2} \Phi_{22}(t, k)+O\left(\varepsilon^{3}\right), \varepsilon \rightarrow 0$, where

$$
\begin{aligned}
\lambda \Phi_{22}(t, k) & =\frac{\alpha_{1}(k) e^{-i t\left(8 k^{3}-1\right)}}{2(2 k-\alpha)}-\frac{\alpha_{1}(k) e^{-i t\left(8 k^{3}+1\right)}}{2\left(2 k+\alpha^{2}\right)}+\frac{\alpha^{2} e^{2 i t}}{8(2 k+\alpha)}-\frac{\alpha e^{-2 i t}}{8\left(2 k-\alpha^{2}\right)}+c_{1}(k) \\
& +\int_{\partial \tilde{D}_{3}^{\sigma k}} c_{2}\left(k, k_{2}\right) e^{-i t\left(8 k_{2}^{3}-1\right)} d k_{2}+\int_{\partial \tilde{D}_{3}^{\sigma k}} c_{3}\left(k, k_{2}\right) e^{-i t\left(8 k_{2}^{3}+1\right)} d k_{2} \\
& +\int_{\partial \tilde{D}_{3}^{\sigma k}} c_{4}\left(k, k_{1}\right) e^{-8 i t\left(k^{3}+k_{1}^{3}\right)} d k_{1}+\int_{\partial \tilde{D}_{3}^{\sigma k}} c_{5}\left(k, k_{1}\right) e^{-i t\left(8 k_{1}^{3}-1\right)} d k_{1} \\
& +\int_{\partial \tilde{D}_{3}^{\sigma k}} c_{6}\left(k, k_{1}\right) e^{-i t\left(8 k_{1}^{3}+1\right)} d k_{1}+\int_{\partial \tilde{D}_{3}^{\tilde{\sigma} k}}\left(\int_{\partial \tilde{D}_{3}^{\sigma k}} c_{7}\left(k, k_{1}, k_{2}\right) e^{-8 i t\left(8 k_{1}^{3}+k_{2}^{3}\right)} d k_{2}\right) d k_{1},
\end{aligned}
$$

with the contour $\tilde{\partial} D_{3}^{\hat{\sigma} k}$ depicted in figure 4 , and the constant $\hat{\sigma}$ is given by

$$
\hat{\sigma}= \begin{cases}1 & \text { if }-k \in \partial D_{3} \\ \alpha & \text { if }-\alpha k \in \partial D_{3} \\ \alpha^{2} & \text { if }-\alpha^{2} k \in \partial D_{3}\end{cases}
$$

The functions $\alpha_{1}(k)$ and $\left\{c_{j}\right\}_{1}^{7}$ are defined by

$$
\begin{aligned}
\alpha_{1}(k) & =-\frac{4 k^{2}\left(\sigma^{2}+\sigma+1\right)}{\left(8 k^{3}+1\right)\left(8 k^{3}-1\right)}, \quad c_{1}(k)=c_{1}^{(1)}(k)+c_{1}^{(2)}(k)+c_{1}^{(3)}(k), \\
c_{2}\left(k, k_{2}\right) & =\frac{(2 k-1)\left(2 k-\alpha^{2}\right)\left(k+k_{2}\right)}{8\left(k^{3}-k_{2}^{3}\right)\left(8 k_{2}^{3}-1\right)} F\left(k_{2}^{3}\right), \\
c_{3}\left(k, k_{2}\right) & =-\frac{(2 k+1)(2 k+\alpha)\left(k+k_{2}\right)}{8\left(k^{3}-k_{2}^{3}\right)\left(8 k_{2}^{3}+1\right)} F\left(k_{2}^{3}\right), \\
c_{4}\left(k, k_{1}\right) & =\frac{\alpha_{1}(k)\left(k-k_{1}\right)}{4\left(k^{3}+k_{1}^{3}\right)} F\left(k_{1}^{3}\right), \quad c_{5}\left(k, k_{1}\right)=-\frac{k-k_{1}}{\left(8 k_{1}^{3}-1\right)(2 k+\alpha)} F\left(k_{1}^{3}\right), \\
c_{6}\left(k, k_{1}\right) & =\frac{k-k_{1}}{\left(8 k_{1}^{3}+1\right)\left(2 k-\alpha^{2}\right)} F\left(k_{1}^{3}\right), \quad c_{7}\left(k, k_{1}, k_{2}\right)=\frac{\left(k-k_{1}\right)\left(k+k_{2}\right)}{16\left(k_{1}^{3}+k_{2}^{3}\right)} F\left(k_{1}^{3}\right) F\left(k_{2}^{3}\right),
\end{aligned}
$$

where

$$
\begin{aligned}
c_{1}^{(1)}(k) & =\frac{2 k(2 \alpha+1)-1}{8\left(4 k^{2}+2 k(2 \alpha+1)-1\right)}+\frac{3 k^{2}-k}{2(\alpha-1)\left(8 k^{3}-1\right)}+\frac{\alpha\left(8 k^{3}+5\right)}{24\left(8 k^{3}-1\right)(2 k-\alpha)} \\
& -\frac{\alpha^{2}\left(8 k^{3}-5\right)}{24\left(8 k^{3}+1\right)\left(2 k+\alpha^{2}\right)}-\frac{\alpha}{4}\left(\frac{8 k^{3}-1}{2\left(8 k^{3}+1\right)}+\frac{\alpha k\left(8 k^{3}-7\right)}{3\left(8 k^{3}-1\right)(2 k-\alpha)}-\frac{\alpha\left(2 k+\alpha^{2}\right)}{6(2 k+\alpha)}-\frac{1}{2}\right) \\
& -\frac{\alpha}{4}\left(\frac{\alpha\left(8 k^{3}+1\right)}{2\left(8 k^{3}-1\right)}+\frac{k\left(8 k^{3}+7\right)}{3\left(8 k^{3}+1\right)\left(2 k+\alpha^{2}\right)}-\frac{2 k-\alpha}{6\left(2 k-\alpha^{2}\right)}-\frac{\alpha}{2}\right) \\
& -\frac{64 k^{5}+8 k^{4}+20 k^{2}+7 k}{12\left(8 k^{3}+1\right)^{2}}-\frac{\alpha\left(3 k^{2}+k\right)}{6\left(8 k^{3}+1\right)}+\frac{112 k^{5}-24 k^{4}-26 k^{2}+9 k}{12\left(8 k^{3}-1\right)^{2}} \\
& +\frac{64 i \sqrt{3} k^{7}+48 i \sqrt{3} k^{5}+16 k^{4}+20 i \sqrt{3} k^{3}+16 k^{2}+1}{6\left(16 k^{4}+4 k^{2}+1\right)^{2}}+\frac{4 i k^{4}\left(3 \log \left(k^{2}\right)-6 \log (k)\right)}{\pi\left(64 k^{6}-1\right)^{2}},
\end{aligned}
$$




$$
\begin{aligned}
c_{1}^{(2)}(k) & =\frac{2 k^{2}\left(\sigma^{2}+\sigma\right)}{\left(64 k^{6}-1\right)\left(2 k+\alpha^{2}\right)(2 k-\alpha)}-\frac{4 \alpha_{1}(k) k^{2}\left(\hat{\sigma}^{2}+\hat{\sigma}+1\right)}{\left(8 k^{3}+1\right)\left(8 k^{3}-1\right)} \\
& -\frac{2 k^{2}\left(\sigma^{2}+\sigma\right)\left(16 k^{4}+8 k^{3}-2 k+1\right)}{\left(8 k^{3}-1\right)^{2}\left(8 k^{3}+1\right)^{2}}-\frac{8 \alpha k^{3}\left(\sigma^{2}+\sigma\right)}{\left(64 k^{6}-1\right)\left(16 k^{4}+4 k^{2}+1\right)},
\end{aligned}
$$

and

$$
c_{1}^{(3)}(k)=\frac{\alpha\left(8 k^{3}-5\right)}{24\left(8 k^{3}-1\right)^{2}}-\frac{\alpha}{8\left(8 k^{3}+1\right)}+\frac{2 k^{3}-1}{3\left(8 k^{3}-1\right)^{2}}-\frac{3}{8(\alpha-1)\left(8 k^{3}-1\right)} .
$$

Proof. Equation (3.21) implies that $\Phi_{22}$ satisfies

$$
\lambda \Phi_{22}(t, k)=\int_{0}^{t}\left[4 k^{2} g_{01}(\tau)-2 i k g_{11}(\tau)-g_{21}(\tau)\right] \Phi_{11}(\tau, k) d \tau+2 i k \int_{0}^{t} g_{01}^{2}(\tau) d \tau
$$

The second integral in (4.23) can be evaluated exactly,

$$
2 i k \int_{0}^{t} g_{01}^{2}(\tau) d \tau=\frac{k}{4}\left(e^{-2 i t}-e^{2 i t}\right)+i k t .
$$

We also note that

$$
\begin{aligned}
4 k^{2} g_{01}(\tau)-2 i k g_{11}(\tau)-g_{21}(\tau)= & -\frac{i}{2}(2 k-1)\left(2 k-\alpha^{2}\right) e^{i \tau}+\frac{i}{2}(2 k+1)(2 k+\alpha) e^{-i \tau} \\
& -2 i \int_{\partial \tilde{D}_{3}}\left(k-k_{1}\right) F\left(k_{1}^{3}\right) e^{-8 i k_{1}^{3} \tau} d k_{1}
\end{aligned}
$$

We denote $\Phi_{11}(t, k)$ by

$$
\Phi_{11}(\tau, k)=\alpha_{1}(k) e^{-8 i k^{3} \tau}+\alpha_{2}(k) e^{i \tau}+\alpha_{3}(k) e^{-i \tau}+\frac{1}{4} \int_{\partial \tilde{D}_{3}^{\sigma k}} \frac{\left(k+k_{2}\right) F\left(k_{2}^{3}\right)}{k^{3}-k_{2}^{3}} e^{-8 i k_{2}^{3} \tau} d k_{2}
$$

where $\alpha_{1}(k)$ is given in (4.17) and $\alpha_{2}(k), \alpha_{3}(k)$ are defined by

$$
\alpha_{2}(k)=-\frac{1}{2(2 k+\alpha)}, \quad \alpha_{3}(k)=\frac{1}{2\left(2 k-\alpha^{2}\right)}
$$

Substituting (4.25) and (4.26) into (4.23) and integrating the resulting expression with respect $d \tau$, we find

$$
\begin{aligned}
\int_{0}^{t}\left[4 k^{2} g_{01}(\tau)-2 i k g_{11}(\tau)-g_{21}(\tau)\right] \Phi_{11}(\tau, k) d \tau=\frac{\alpha_{1}(k)}{2(2 k-\alpha)}\left(e^{-i t\left(8 k^{3}-1\right)}-1\right) \\
\quad-\frac{\alpha_{1}(k)}{2\left(2 k+\alpha^{2}\right)}\left(e^{-i t\left(8 k^{3}+1\right)}-1\right)-\frac{\alpha_{2}(k)}{4}(2 k-1)\left(2 k-\alpha^{2}\right)\left(e^{2 i t}-1\right) \\
-\frac{\alpha_{3}(k)}{4}(2 k+1)(2 k+\alpha)\left(e^{-2 i t}-1\right)+\frac{i \alpha_{2}(k)}{2}(2 k+1)(2 k+\alpha) t \\
-\frac{i \alpha_{3}(k)}{2}(2 k-1)\left(2 k-\alpha^{2}\right) t+I(t, k)
\end{aligned}
$$


where

$$
\begin{aligned}
I(t, k) & =\frac{1}{8}(2 k-1)\left(2 k-\alpha^{2}\right) \int_{\partial \tilde{D}_{3}^{\sigma k}} \frac{\left(k+k_{2}\right)\left(e^{-i t\left(8 k_{2}^{3}-1\right)}-1\right)}{\left(k^{3}-k_{2}^{3}\right)\left(8 k_{2}^{3}-1\right)} F\left(k_{2}^{3}\right) d k_{2} \\
& -\frac{1}{8}(2 k+1)(2 k+\alpha) \int_{\partial \tilde{D}_{3}^{\sigma k}} \frac{\left(k+k_{2}\right)\left(e^{-i t\left(8 k_{2}^{3}+1\right)}-1\right)}{\left(k^{3}-k_{2}^{3}\right)\left(8 k_{2}^{3}+1\right)} F\left(k_{2}^{3}\right) d k_{2} \\
& +2 \alpha_{2}(k) \int_{\partial \tilde{D}_{3}} \frac{\left(k-k_{1}\right)\left(e^{-i t\left(8 k_{1}^{3}-1\right)}-1\right)}{8 k_{1}^{3}-1} F\left(k_{1}^{3}\right) d k_{1} \\
& +2 \alpha_{3}(k) \int_{\partial \tilde{D}_{3}} \frac{\left(k-k_{1}\right)\left(e^{-i t\left(8 k_{1}^{3}+1\right)}-1\right)}{8 k_{1}^{3}+1} F\left(k_{1}^{3}\right) d k_{1} \\
& +\frac{\alpha_{1}(k)}{4} \int_{\partial \tilde{D}_{3}} \frac{\left(k-k_{1}\right)\left(e^{-8 i t\left(k^{3}+k_{1}^{3}\right)}-1\right)}{k^{3}+k_{1}^{3}} F\left(k_{1}^{3}\right) d k_{1} \\
& +\frac{1}{16} \int_{\partial \tilde{D}_{3}}\left(\int_{\partial \tilde{D}_{3}^{\sigma k}} \frac{\left(k-k_{1}\right)\left(k+k_{2}\right)\left(e^{-8 i t\left(k_{1}^{3}+k_{2}^{3}\right)}-1\right)}{\left(k^{3}-k_{2}^{3}\right)\left(k_{1}^{3}+k_{2}^{3}\right)} F\left(k_{1}^{3}\right) F\left(k_{2}^{3}\right) d k_{2}\right) d k_{1} .
\end{aligned}
$$

Using the definitions for $\alpha_{2}(k)$ and $\alpha_{3}(k)$ in (4.27), we find

$$
\frac{i \alpha_{2}(k)}{2}(2 k+1)(2 k+\alpha) t-\frac{i \alpha_{3}(k)}{2}(2 k-1)\left(2 k-\alpha^{2}\right) t=-i k t ;
$$

thus, the term in (4.24) involving $t$ cancels and then employing (4.24), equation (4.23) becomes

$$
\begin{aligned}
\lambda \Phi_{22}(t, k) & =\frac{\alpha_{1}(k)}{2(2 k-\alpha)}\left(e^{-i t\left(8 k^{3}-1\right)}-1\right)-\frac{\alpha_{1}(k)}{2\left(2 k+\alpha^{2}\right)}\left(e^{-i t\left(8 k^{3}+1\right)}-1\right) \\
& -\frac{\alpha^{2} e^{2 i t}}{8(2 k+\alpha)}-\frac{\alpha e^{-2 i t}}{8\left(2 k-\alpha^{2}\right)}+\frac{2 k(2 \alpha+1)-1}{8\left(4 k^{2}+2 k(2 \alpha+1)-1\right)}+I(t, k) .
\end{aligned}
$$

In what follows we evaluate each integral appearing in $I(t, k)$. Using the residue theorem we find

$$
\begin{aligned}
\int_{\partial \tilde{D}_{3}^{\sigma k}} \frac{\left(k+k_{2}\right) F\left(k_{2}^{3}\right)}{\left(k^{3}-k_{2}^{3}\right)\left(8 k_{2}^{3}-1\right)} d k_{2}= & \frac{\alpha(2 k-\alpha)}{8 k^{3}+1}+\frac{2 \alpha^{2} k\left(8 k^{3}-7\right)}{3\left(8 k^{3}-1\right)^{2}}-\frac{\alpha\left(8 k^{3}+5\right)}{3\left(8 k^{3}-1\right)^{2}} \\
& +\frac{16 k^{2}\left(\sigma^{2}+\sigma\right)}{\left(8 k^{3}-1\right)^{2}\left(8 k^{3}+1\right)}:=\beta_{1}(k), \\
\int_{\partial \tilde{D}_{3}^{\sigma k}} \frac{\left(k+k_{2}\right) F\left(k_{2}^{3}\right)}{\left(k^{3}-k_{2}^{3}\right)\left(8 k_{2}^{3}+1\right)} d k_{2}= & -\frac{\alpha^{2}\left(2 k+\alpha^{2}\right)}{8 k^{3}-1}-\frac{2 \alpha k\left(8 k^{3}+7\right)}{3\left(8 k^{3}+1\right)^{2}}-\frac{\alpha^{2}\left(8 k^{3}-5\right)}{3\left(8 k^{3}+1\right)^{2}} \\
& +\frac{16 k^{2}\left(\sigma^{2}+\sigma\right)}{\left(8 k^{3}-1\right)\left(8 k^{3}+1\right)^{2}}:=\beta_{2}(k), \\
\int_{\partial \tilde{D}_{3}} \frac{\left(k-k_{1}\right) F\left(k_{1}^{3}\right)}{8 k_{1}^{3}-1} d k_{1}= & \frac{\alpha(2 k+\alpha)}{8}+\frac{\alpha^{2}\left(2 k+\alpha^{2}\right)}{24}, \\
\int_{\partial \tilde{D}_{3}} \frac{\left(k-k_{1}\right) F\left(k_{1}^{3}\right)}{8 k_{1}^{3}-1} d k_{1}= & -\frac{\alpha^{2}\left(2 k-\alpha^{2}\right)}{8}-\frac{\alpha(2 k-\alpha)}{24} .
\end{aligned}
$$


We note that the integrand in the fifth line in (4.29) has removable singularities at $k_{1}=$ $-k,-\alpha k,-\alpha^{2} k$ and hence before splitting the integral we deform $\partial \tilde{D}_{3}$ to $\partial \tilde{D}_{3}^{\hat{\sigma} k}$ depicted in figure 4. Using the residue theorem we find

$$
\int_{\partial \tilde{D}_{3}} \frac{\left(k-k_{1}\right) F\left(k_{1}^{3}\right)}{k^{3}+k_{1}^{3}} d k_{1}=-\frac{2 \alpha(2 k+\alpha)}{8 k^{3}-1}-\frac{2 \alpha^{2}\left(2 k-\alpha^{2}\right)}{8 k^{3}+1}+\frac{16 k^{2}\left(\hat{\sigma}^{2}+\hat{\sigma}\right)}{\left(8 k^{3}+1\right)\left(8 k^{3}-1\right)}:=\beta_{3}(k),
$$

where the constant $\hat{\sigma}$ is defined in (4.16).

Regarding the double integral, we deform $\partial \tilde{D}_{3}$ to $\partial \tilde{D}_{3}^{\tilde{\sigma} k_{2}}$, where $\tilde{\sigma}$ is defined by

$$
\tilde{\sigma}= \begin{cases}1 & \text { if }-k_{2} \in \partial D_{3} \\ \alpha & \text { if }-\alpha k_{2} \in \partial D_{3} \\ \alpha^{2} & \text { if }-\alpha^{2} k_{2} \in \partial D_{3}\end{cases}
$$

Then, using a residue calculation we find

$$
\int_{\partial \tilde{D}_{3}^{\tilde{\sigma} k_{2}}} \frac{\left(k-k_{1}\right) F\left(k_{1}^{3}\right)}{k_{1}^{3}+k_{2}^{3}} d k_{1}=-\frac{2 \alpha(2 k+\alpha)}{8 k_{2}^{3}-1}-\frac{2 \alpha^{2}\left(2 k-\alpha^{2}\right)}{8 k_{2}^{3}+1}+\frac{16 \tilde{\sigma} k_{2}\left(k+\tilde{\sigma} k_{2}\right)}{\left(8 k_{2}^{3}+1\right)\left(8 k_{2}^{3}-1\right)} .
$$

Thus, the double integral denoted by $\beta_{4}(k)$ can be written as

$$
\begin{aligned}
\beta_{4}(k) & =-\frac{1}{16} \int_{\partial \tilde{D}_{3}^{\sigma k}}\left(\int_{\partial \tilde{D}_{3}^{\tilde{\sigma} k_{2}}} \frac{\left(k-k_{1}\right)\left(k+k_{2}\right)}{\left(k^{3}-k_{2}^{3}\right)\left(k_{1}^{3}+k_{2}^{3}\right)} F\left(k_{1}^{3}\right) F\left(k_{2}^{3}\right) d k_{1}\right) d k_{2} \\
& =\beta_{4}^{(1)}(k)+\beta_{4}^{(2)}(k)+\beta_{4}^{(3)}(k),
\end{aligned}
$$

with

and

$$
\begin{aligned}
& \beta_{4}^{(1)}(k)=-\int_{C_{1}^{\sigma k}} \frac{\alpha k_{2}\left(k+k_{2}\right)\left(k+\alpha k_{2}\right) F\left(k_{2}^{3}\right)}{\left(k^{3}-k_{2}^{3}\right)\left(8 k_{2}^{3}+1\right)\left(8 k_{2}^{3}-1\right)} d k_{2}, \\
& \beta_{4}^{(2)}(k)=-\int_{C_{2}^{\sigma k}} \frac{\alpha^{2} k_{2}\left(k+k_{2}\right)\left(k+\alpha^{2} k_{2}\right) F\left(k_{2}^{3}\right)}{\left(k^{3}-k_{2}^{3}\right)\left(8 k_{2}^{3}+1\right)\left(8 k_{2}^{3}-1\right)} d k_{2},
\end{aligned}
$$

$$
\beta_{4}^{(3)}(k)=\frac{1}{8} \int_{\partial \tilde{D}_{3}^{\sigma k}} \frac{\left(k+k_{2}\right) F\left(k_{2}^{3}\right)}{k^{3}-k_{2}^{3}}\left[\frac{\alpha(2 k+\alpha)}{8 k_{2}^{3}-1}+\frac{\alpha^{2}\left(2 k-\alpha^{2}\right)}{8 k_{2}^{3}+1}\right] d k_{2},
$$

where $C_{1}^{\sigma k}$ and $C_{2}^{\sigma k}$ are depicted in figure 4 . A residue calculation implies

$$
\begin{aligned}
\beta_{4}^{(3)}(k) & =-\frac{128 k^{5}+16 k^{4}-8 k^{3}+40 k^{2}+14 k+5}{24\left(8 k^{3}+1\right)^{2}}-\frac{\alpha\left(12 k^{2}+4 k+3\right)}{24\left(8 k^{3}+1\right)^{2}} \\
& +\frac{112 k^{5}-24 k^{4}+8 k^{3}-26 k^{2}+9 k-4}{12\left(8 k^{3}-1\right)^{2}}+\frac{12 k^{2}-4 k-3}{8(\alpha-1)\left(8 k^{3}-1\right)} \\
& -\frac{2 k^{2}\left(\sigma^{2}+\sigma\right)\left(16 k^{4}+8 k^{3}-2 k+1\right)}{\left(64 k^{6}-1\right)^{2}}-\frac{8 \alpha k^{2}\left(\sigma^{2}+\sigma\right)}{\left(64 k^{6}-1\right)\left(16 k^{4}+4 k^{2}+1\right)} .
\end{aligned}
$$

In order to compute $\beta_{4}^{(1)}$ by Cauchy's theorem, we first deform $C_{1}$ to $\tilde{C}_{1}$ (cf. figure 2 ). Using $k_{2}=r v$ with $v=e^{-i \pi / 6}, \beta_{4}^{(1)}(k)$ can be written in the form

$$
\beta_{4}^{(1)}(k)=\frac{12}{i \pi} \int_{0}^{\infty} \frac{\alpha r^{4} v^{5}(k+r v)(k+\alpha r v)}{\left(k^{3}-r^{3} v^{3}\right)\left(8 r^{3} v^{3}+1\right)\left(8 r^{3} v^{3}-1\right)}\left(\frac{1}{8 r^{3} v^{3}+1}-\frac{1}{8 r^{3} v^{3}-1}\right) d r .
$$


For $\beta_{4}^{(2)}(k)$, letting $k_{2} \rightarrow \alpha^{2} k_{2}$, in analogy with $\beta_{4}^{(1)}(k)$, we obtain

$$
\beta_{4}^{(2)}(k)=-\frac{12}{i \pi} \int_{0}^{\infty} \frac{\alpha r^{4} v^{5}(k+\alpha r v)\left(k+\alpha^{2} r v\right)}{\left(k^{3}-r^{3} v^{3}\right)\left(8 r^{3} v^{3}+1\right)\left(8 r^{3} v^{3}-1\right)}\left(\frac{1}{8 r^{3} v^{3}+1}-\frac{1}{8 r^{3} v^{3}-1}\right) d r .
$$

Evaluating (4.44) and (4.45) directly, we find

$$
\begin{aligned}
\beta_{4}^{(1)}(k)+\beta_{4}^{(2)}(k) & =\frac{64 i \sqrt{3} k^{7}+48 i \sqrt{3} k^{5}+16 k^{4}+20 i \sqrt{3} k^{3}+16 k^{2}+1}{6(2 k+\alpha)^{2}\left(2 k+\alpha^{2}\right)^{2}(2 k-\alpha)^{2}\left(2 k-\alpha^{2}\right)^{2}} \\
& +\frac{4 i k^{4}\left(3 \log \left(k^{2}\right)-6 \log (k)\right)}{\pi\left(64 k^{6}-1\right)^{2}} .
\end{aligned}
$$

Combining the above results, we find (4.15), where $c_{1}(k)$ is given by

$$
\begin{aligned}
c_{1}(k) & =\frac{\alpha_{1}(k)}{2\left(2 k+\alpha^{2}\right)(2 k-\alpha)}+\frac{2 k(2 \alpha+1)-1}{8\left(4 k^{2}+2 k(2 \alpha+1)-1\right)}+\frac{\alpha^{2}\left(2 k+\alpha^{2}\right)}{24(2 k+\alpha)}+\frac{\alpha(2 k-\alpha)}{24\left(2 k-\alpha^{2}\right)}-\frac{1}{8} \\
& -\frac{1}{8}(2 k-1)\left(2 k-\alpha^{2}\right) \beta_{1}(k)+\frac{1}{8}(2 k+1)(2 k+\alpha) \beta_{2}(k)-\frac{\alpha_{1}(k)}{4} \beta_{3}(k)+\beta_{4}(k) .
\end{aligned}
$$

Simplifying (4.47), we find that $c_{1}(k)$ is given by the second equation in (4.17), where $\left\{c_{1}^{(j)}\right\}_{1}^{3}$ are defined in (4.22).

Proposition 4.4. Let $q(x, 0)$ and $q(0, t)$ be given by (4.1). Then, $q_{x}(0, t)$ and $q_{x x}(0, t)$ are given by (3.3) and (3.7), where $g_{11}, g_{12}, g_{21}, g_{22}$ are given in proposition 4.1 and $g_{13}, g_{23}$ are given by the following formulas:

$$
\begin{aligned}
\lambda g_{13}(t)= & \left(\frac{\alpha^{2}}{16}-\frac{\alpha^{2}}{32}\left(3^{1 / 3}-1\right)\right) e^{3 i t}+\left(\frac{\alpha}{16}-\frac{\alpha}{32}\left(3^{1 / 3}-1\right)\right) e^{-3 i t} \\
& +d_{1} e^{i t}+d_{2} e^{-i t}+o(1), \quad t \rightarrow \infty
\end{aligned}
$$

and

$$
\begin{aligned}
\lambda g_{23}(t)= & \left(\frac{i}{4}-\frac{i}{32}\left(3^{2 / 3}-1\right)\right) e^{3 i t}-\left(\frac{i}{4}-\frac{i}{32}\left(3^{2 / 3}-1\right)\right) e^{-3 i t} \\
& +d_{3} e^{i t}+d_{4} e^{-i t}+o(1), \quad t \rightarrow \infty,
\end{aligned}
$$

with $d_{1}, \ldots, d_{4}$ constants.

Proof. We will derive equation (4.48); equation (4.49) can be derived in a similar way. Recalling (3.20), we denote $\lambda g_{13}(t)$ by

$$
\lambda g_{13}(t)=\lambda g_{13}^{(1)}(t)+\lambda g_{13}^{(2)}(t)
$$

where

$$
\begin{aligned}
& \lambda g_{13}^{(1)}(t)=\frac{2}{i \pi} \int_{\partial D_{3}} k \lambda\left[\Phi_{13}(t, k)+\alpha \Phi_{13}(t, \alpha k)+\alpha^{2} \Phi_{13}\left(t, \alpha^{2} k\right)\right] d k \\
& \lambda g_{13}^{(2)}(t)=\frac{e^{i t}-e^{-i t}}{\pi} \int_{\partial D_{3}} \lambda\left[\Phi_{22}(t, k)+\alpha \Phi_{22}(t, \alpha k)+\alpha^{2} \Phi_{22}\left(t, \alpha^{2} k\right)\right] d k .
\end{aligned}
$$

We next compute the coefficients of the terms involving $e^{3 i t}$ and $e^{-3 i t}$. 
Regarding $\lambda g_{13}^{(2)}(t)$, we use (4.15). Note that the term involving $e^{3 i t}$ arises from the third term in (4.15),

$$
\frac{\alpha^{2} e^{2 i t}}{8(2 k+\alpha)}
$$

the relevant contribution for $\lambda g_{13}^{(2)}(t)$ is given by

$$
\frac{\alpha^{2}}{8 i \pi} \int_{\partial \tilde{D}_{3}}\left(\frac{1}{2 k+\alpha}+\frac{\alpha}{2 \alpha k+\alpha}+\frac{\alpha^{2}}{2 \alpha^{2} k+\alpha}\right) d k=\frac{3 \alpha^{2}}{2 i \pi} \int_{\partial \tilde{D}_{3}} \frac{k^{2}}{8 k^{3}+1} d k=\frac{\alpha^{2}}{16},
$$

where we have used the fact that the integrand has poles at $k=-\alpha / 2$ and at $k=\infty$. Also, noting that the term involving $e^{-3 i t}$ arises from the fourth term in (4.15),

$$
\frac{\alpha e^{-2 i t}}{8\left(2 k-\alpha^{2}\right)}
$$

the relevant contribution for $\lambda g_{13}^{(2)}(t)$ is given by

$$
\frac{\alpha}{8 i \pi} \int_{\partial \tilde{D}_{3}}\left(\frac{1}{2 k-\alpha^{2}}+\frac{\alpha}{2 \alpha k-\alpha^{2}}+\frac{\alpha^{2}}{2 \alpha^{2} k-\alpha^{2}}\right) d k=\frac{3 \alpha^{2}}{2 i \pi} \int_{\partial \tilde{D}_{3}} \frac{k^{2}}{8 k^{3}-1} d k=\frac{\alpha}{16},
$$

where we have used the fact that the integrand has poles at $k=\alpha^{2} / 2$ and at $k=\infty$.

Regarding $\lambda g_{13}^{(1)}(t)$, we use (3.24). According to the symmetric combination for $\lambda \Phi_{13}(t, k)$ in (4.51), we first need to evaluate the integrals

$$
\begin{aligned}
-2 i k e^{-8 i k^{3} t} & \int_{0}^{t} e^{8 i k^{3} \tau} g_{01}^{2}(\tau) \Phi_{11}(\tau, k) d \tau \\
& +e^{-8 i k^{3} t} \int_{0}^{t} e^{8 i k^{3} \tau}\left[4 k^{2} g_{01}(\tau)+2 i k g_{11}(\tau)-g_{21}(\tau)\right] \Phi_{22}(\tau, k) d \tau
\end{aligned}
$$

For the first integral (involving $\Phi_{11}(\tau, k)$ ), we substitute (4.26) into (4.57) and then integrate the resulting expression with respect $d \tau$; in this way we find the following explicit expressions:

$$
\begin{aligned}
& \frac{2}{k} e^{-8 i k^{3} t}\left[-2 i \alpha_{1}(k) t+\frac{\alpha_{1}(k)\left(e^{2 i t}-1\right)}{2}-\frac{\alpha_{1}(k)\left(e^{-2 i t}-1\right)}{2}+\left(\alpha_{3}(k)-2 \alpha_{2}(k)\right) \frac{e^{i t\left(8 k^{3}+1\right)}-1}{8 k^{3}+1}\right. \\
& \left.\quad+\left(\alpha_{2}(k)-2 \alpha_{3}(k)\right) \frac{e^{i t\left(8 k^{3}-1\right)}-1}{8 k^{3}-1}+\frac{\alpha_{2}(k)\left(e^{i t\left(8 k^{3}+3\right)}-1\right)}{8 k^{3}+3}+\frac{\alpha_{3}(k)\left(e^{i t\left(8 k^{3}-3\right)}-1\right)}{8 k^{3}-3}\right] .
\end{aligned}
$$

Hence, the term involving $e^{3 i t}$ arises from the term

$$
\frac{k \alpha_{2}(k)}{2\left(8 k^{3}+3\right)}=-\frac{k}{4(2 k+\alpha)\left(8 k^{3}+3\right)} .
$$

The relevant contribution for $\lambda g_{13}^{(1)}(t)$ is given by

$$
-\frac{1}{2 i \pi} \int_{\partial \tilde{D}_{3}} \frac{k}{8 k^{3}+3}\left(\frac{k}{2 k+\alpha}+\frac{\alpha^{2} k}{2 \alpha k+\alpha}+\frac{\alpha k}{2 \alpha^{2} k+\alpha}\right) d k=\frac{3 \alpha}{i \pi} \int_{\partial \tilde{D}_{3}} \frac{k^{3}}{\left(8 k^{3}+3\right)\left(8 k^{3}+1\right)} d k .
$$


Note that the above integrand has poles at $k=-\alpha / 2$ and $k=-\frac{3^{\frac{1}{3}}}{2} \alpha$; thus,

$$
\frac{3 \alpha}{i \pi} \int_{\partial \tilde{D}_{3}} \frac{k^{3}}{\left(8 k^{3}+3\right)\left(8 k^{3}+1\right)} d k=\frac{\alpha^{2}}{16}\left(3^{1 / 3}-1\right) .
$$

The term involving $e^{-3 i t}$ arises from the term

$$
\frac{k \alpha_{3}(k)}{2\left(8 k^{3}-3\right)}=\frac{k}{4\left(2 k-\alpha^{2}\right)\left(8 k^{3}-3\right)},
$$

and the relevant contribution for $\lambda g_{13}^{(1)}(t)$ is given by

$$
\begin{aligned}
& \frac{1}{2 i \pi} \int_{\partial \tilde{D}_{3}} \frac{k}{8 k^{3}-3}\left(\frac{k}{2 k-\alpha^{2}}+\frac{\alpha^{2} k}{2 \alpha k-\alpha^{2}}+\frac{\alpha k}{2 \alpha^{2} k-\alpha^{2}}\right) d k \\
& \quad=\frac{3 \alpha}{i \pi} \int_{\partial \tilde{D}_{3}} \frac{k^{3}}{\left(8 k^{3}-3\right)\left(8 k^{3}-1\right)} d k .
\end{aligned}
$$

The above integrand has poles at $k=\alpha^{2} / 2$ and $k=\frac{3^{\frac{1}{3}}}{2} \alpha^{2}$, thus

$$
\frac{3 \alpha}{i \pi} \int_{\partial \tilde{D}_{3}} \frac{k^{3}}{\left(8 k^{3}+3\right)\left(8 k^{3}+1\right)} d k=\frac{\alpha}{16}\left(3^{1 / 3}-1\right) .
$$

For the second integral in (4.57) (involving $\Phi_{22}(\tau, k)$ ), we substitute (4.15) into (4.57). We need to evaluate the integral

$$
\begin{aligned}
& e^{-8 i k^{3} t} \int_{0}^{t}\left[-\frac{i}{2} e^{i \tau}\left(4 k^{2}-2 \alpha k+\alpha^{2}\right)+\frac{i}{2} e^{-i \tau}\left(4 k^{2}+2 \alpha^{2} k+\alpha\right)\right] \\
& \quad \times\left[\frac{\alpha_{1}(k) e^{i \tau}}{2(2 k-\alpha)}-\frac{\alpha_{1}(k) e^{-i \tau}}{2\left(2 k+\alpha^{2}\right)}+\frac{\alpha^{2} e^{i \tau\left(8 k^{3}+2\right)}}{8(2 k+\alpha)}-\frac{\alpha e^{i \tau\left(8 k^{3}-2\right)}}{8\left(2 k-\alpha^{2}\right)}+c_{1}(k) e^{8 i k^{3} \tau}\right] d \tau .
\end{aligned}
$$

Letting

$$
u_{1}(k)=4 k^{2}-2 \alpha k+\alpha^{2}, \quad u_{2}(k)=4 k^{2}+2 \alpha^{2} k+\alpha,
$$

and integrating the above expression with respect $d \tau$, equation (4.65) can be written in the form

$$
\begin{aligned}
& e^{-8 i k^{3} t}\left[\frac{i \alpha t}{4}\left(\frac{u_{1}(k)}{2 k+\alpha^{2}}+\frac{u_{2}(k)}{2 k-\alpha}\right)-\frac{\alpha_{1}(k) u_{1}(k)\left(e^{2 i t}-1\right)}{8(2 k-\alpha)}+\frac{\alpha_{1}(k) u_{2}(k)\left(e^{-2 i t}-1\right)}{8\left(2 k+\alpha^{2}\right)}\right. \\
& -\frac{e^{i t\left(8 k^{3}+1\right)}-1}{2\left(8 k^{3}+1\right)}\left(c_{1}(k) u_{1}(k)-\frac{\alpha^{2} u_{2}(k)}{8(2 k+\alpha)}\right)+\frac{e^{i t\left(8 k^{3}-1\right)}-1}{2\left(8 k^{3}-1\right)}\left(c_{1}(k) u_{2}(k)+\frac{\alpha u_{1}(k)}{8\left(2 k-\alpha^{2}\right)}\right) \\
& \left.-\frac{\alpha^{2} u_{1}(k)\left(e^{i t\left(8 k^{3}+3\right)}-1\right)}{16\left(8 k^{3}+3\right)(2 k+\alpha)}-\frac{\alpha u_{2}(k)\left(e^{i t\left(8 k^{3}-3\right)}-1\right)}{16\left(8 k^{3}-3\right)\left(2 k-\alpha^{2}\right)}\right] .
\end{aligned}
$$

Thus, the term involving $e^{3 i t}$ arises from the term

$$
-\frac{\alpha^{2} u_{1}(k)}{16\left(8 k^{3}+3\right)(2 k+\alpha)} .
$$


The relevant contribution for $\lambda g_{13}^{(1)}(t)$ is given by

$$
\begin{aligned}
-\frac{\alpha^{2}}{8 i \pi} & \int_{\partial \tilde{D}_{3}} \frac{k}{8 k^{3}+3}\left(\frac{u_{1}(k)}{2 k+\alpha}+\frac{\alpha u_{1}(\alpha k)}{2 \alpha k+\alpha}+\frac{\alpha^{2} u_{1}\left(\alpha^{2} k\right)}{2 \alpha^{2} k+\alpha}\right) d k \\
& =-\frac{9 \alpha}{2 i \pi} \int_{\partial \tilde{D}_{3}} \frac{k^{3}}{\left(8 k^{3}+3\right)\left(8 k^{3}+1\right)} d k=-\frac{3 \alpha^{2}}{32}\left(3^{1 / 3}-1\right),
\end{aligned}
$$

where we have used the fact that the integrand has poles at $k=-\alpha / 2$ and $k=-\frac{3^{\frac{1}{3}}}{2} \alpha$. Similarly, we find that the term involving $e^{-3 i t}$ arises from the term

$$
-\frac{\alpha u_{2}(k)}{16\left(8 k^{3}-3\right)\left(2 k-\alpha^{2}\right)}
$$

The relevant contribution for $\lambda g_{13}^{(1)}(t)$ is given by

$$
\begin{array}{r}
-\frac{\alpha}{8 i \pi} \int_{\partial \tilde{D}_{3}} \frac{k}{8 k^{3}-3}\left(\frac{u_{2}(k)}{2 k-\alpha^{2}}+\frac{\alpha u_{2}(\alpha k)}{2 \alpha k-\alpha^{2}}+\frac{\alpha^{2} u_{2}\left(\alpha^{2} k\right)}{2 \alpha^{2} k-\alpha^{2}}\right) d k \\
=-\frac{9 \alpha^{2}}{2 i \pi} \int_{\partial \tilde{D}_{3}} \frac{k^{3}}{\left(8 k^{3}-3\right)\left(8 k^{3}-1\right)} d k=-\frac{3 \alpha}{32}\left(3^{1 / 3}-1\right),
\end{array}
$$

where we have used the fact that the integrand has poles at $k=\alpha^{2} / 2$ and $k=\frac{3^{\frac{1}{3}}}{2} \alpha^{2}$.

Combining (4.54), (4.61) and (4.69) for the terms involving $e^{3 i t}$, as well as (4.56), (4.64) and (4.71) for the terms involving $e^{-3 i t}$, we find the following expressions for $e^{3 i t}$ and $e^{-3 i t}$ :

$$
\left(\frac{\alpha^{2}}{16}-\frac{\alpha^{2}}{32}\left(3^{1 / 3}-1\right)\right) e^{3 i t}+\left(\frac{\alpha}{16}-\frac{\alpha}{32}\left(3^{1 / 3}-1\right)\right) e^{-3 i t}
$$

\section{Acknowledgments}

The authors are grateful to the EPSRC, UK, for partial support.

\section{References}

[1] A. de Monvel Boutet, A. S. Fokas and D. Shepelsky, Analysis of the global relation for the nonlinear Schrödinger equation on the half-line, Lett. Math. Phys. 65 (2003) 199-212.

[2] A. de Monvel Boutet, A. Its and V. Kotlyarov, Long-time asymptotics for the focusing NLS equation with time-periodic boundary condition, C. R. Math. Acad. Sci. Paris 345 (2007) 615-620.

[3] A. de Monvel Boutet, A. Its, V. Kotlyarov, Long-time asymptotics for the focusing NLS equation with time-periodic boundary condition on the half-line, Commun. Math. Phys. 290 (2009) 479-522.

[4] A. de Monvel Boutet, A. Kotlyarov, D. Shepelsky and C. Zheng, Initial boundary value problems for integrable systems: towards the long-time asymptotics, Nonlinearity 23 (2010) 2483.

[5] P. Deift, S. Venakides and X. Zhou, The collisionless shock region for the long time behavior of the solutions of the KdV equation, Commun. Pure Appl. Math. 47 (1994) 199-206.

[6] P. Deift, S. Venakides and X. Zhou, New results in small dispersion KdV by an extension of the steepest descent method for Riemann-Hilbert problems, Int. Math. Res. Notices 6 (1997) 286-299.

[7] P. Deift and X. Zhou, A steepest descent method for oscillatory Riemann-Hilbert problems, Bull. Amer. Math. Soc. 26 (1992) 119-123. 
[8] P. Deift and X. Zhou, A steepest descent method for oscillatory Riemann-Hilbert problems. Asymptotics for the mKdV equation, Ann. Math. 137 (1993) 295-368.

[9] A. S. Fokas, A unified transform method for solving linear and certain nonlinear PDEs, Proc. Roy. Soc. London A 453 (1997) 1411-1443.

[10] A S Fokas, On the integrability of certain linear and nonlinear partial differential equations, J. Math. Phys. 41 (2000) 4188-4237.

[11] A. S. Fokas, Integrable nonlinear evolution equations on the half-line, Comm. Math. Phys. 230 (2002) 1-39.

[12] A. S. Fokas, The generalized Dirichlet-to-Neumann map for certain nonlinear evolution PDEs, Comm. Pure Appl. Math. LVIII (2005) 639-670.

[13] A. S. Fokas, Lax pairs: novel type of separability, Inv. Probl. 25 (2009) 123007.

[14] A. S. Fokas and G. Hwang, An example of an explicit construction of the asymptotic form of the Neumann boundary value for the NLS, Preprint.

[15] A. S. Fokas and J. Lenells, The unified method: I. Non-linearizable problems on the half-line, J. Phys. A: Math. Theor. 45 (2012) 195201.

[16] A. S. Fokas A.S and J. Lenells, The unified method: IV. The explicit form of the Neumann boundary value for the NLS, in preparation.

[17] A. S. Fokas and E. A. Spence, Synthesis, as opposed to separation, of variables, SIAM Review 25 (2012) 291-324.

[18] J. Lenells and A. S. Fokas, The unified method on the half-line: II. NLS on the half-line with $t$-periodic boundary conditions, J. Phys. A: Math. Theor. 45 (2012) 195202. 